TI 2017-101/II

Tinbergen Institute Discussion Paper

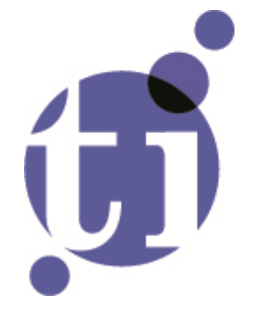

\title{
Dynamic Interbank Network Analysis Using Latent Space Models
}

Fernando Linardi ${ }^{1}$

Cees (C.G.H.) Diks²

Marco (M.J.) van der Leij ${ }^{3}$

luri Lazier ${ }^{4}$

1: University of Amsterdam, The Netherlands; Central Bank of Brazil, Brazil

2: University of Amsterdam, The Netherlands; Tinbergen Institute, The Netherlands

3: University of Amsterdam, The Netherlands; De Nederlandsche Bank, The Netherlands

4: Central Bank of Brazil, Brazil 
Tinbergen Institute is the graduate school and research institute in economics of Erasmus University Rotterdam, the University of Amsterdam and VU University Amsterdam.

Contact: discussionpapers@tinbergen.nl

More TI discussion papers can be downloaded at the Tinbergen Site

Tinbergen Institute has two locations:

Tinbergen Institute Amsterdam

Gustav Mahlerplein 117

1082 MS Amsterdam

The Netherlands

Tel.: +31(0)20 5984580

Tinbergen Institute Rotterdam

Burg. Oudlaan 50

3062 PA Rotterdam

The Netherlands

Tel.: +31(0)10408 8900 


\title{
Dynamic interbank network analysis using latent space models
}

\author{
Fernando Linardi ${ }^{1}$ Cees Diks ${ }^{2} \quad$ Marco van der Leij ${ }^{3} \quad$ Iuri Lazier ${ }^{4}$ \\ September 14, 2017
}

\begin{abstract}
Longitudinal network data are increasingly available, allowing researchers to model how networks evolve over time and to make inference on their dependence structure. In this paper, a dynamic latent space approach is used to model directed networks of monthly interbank exposures. In this model, each node has an unobserved temporal trajectory in a low-dimensional Euclidean space. Model parameters and latent banks' positions are estimated within a Bayesian framework. We apply this methodology to analyze two different datasets: the unsecured and the secured (repo) interbank lending networks. We show that the model that incorporates a latent space performs much better than the model in which the probability of a tie depends only on observed characteristics; the latent space model is able to capture some features of the dyadic data such as transitivity that the model without a latent space is not able to.
\end{abstract}

Keywords: network dynamics, latent position model, interbank network, Bayesian inference.

JEL Classification: C11, D85, G21

\footnotetext{
${ }^{1}$ University of Amsterdam and Central Bank of Brazil. e-mail: fernando.linardi@bcb.gov.br

${ }^{2}$ CeNDEF, University of Amsterdam and Tinbergen Institute. e-mail: C.G.H.Diks@uva.nl

${ }^{3}$ CeNDEF, University of Amsterdam, Tinbergen Institute and Research Department, De Nederlandsche Bank. e-mail: M.J.vanderLeij@uva.nl

${ }^{4}$ Central Bank of Brazil. e-mail: iuri.lazier@bcb.gov.br

The opinions expressed in this article are those of the authors and do not necessarily reflect the views of the Central Bank of Brazil or De Nederlandsche Bank.
} 


\section{Introduction}

There has been a growing interest in the study of networks in economics over the last years. Specifically in finance the accelerated loss of confidence in financial markets following the failure of Lehman Brothers in 2008 has shown the importance of understanding the network of linkages between financial institutions. The lack of knowledge of counterparty exposures and how the contagion would spread throughout the system during the crisis have led policy makers to adopt a large set of financial reforms to address these and other vulnerabilities, both at the international and domestic level (Claessens and Kodres, 2014).

Since financial institutions are highly interconnected, network theory provides a useful framework for the analysis of the financial system. Interconnections may be directed due to banks' claims on each other or may arise indirectly when banks hold similar portfolios or share a common pool of investors or depositors. The role of these interconnections in the propagation of shocks in financial networks has been analyzed by a growing literature. Allen and Gale (2000) and Freixas et al. (2000) have shown how contagion depends on the structure of the interbank network. In more recent work, Acemoglu et al. (2015) show that for a small shock hitting the system a more connected financial network enhances financial stability. On the other hand, a less connected financial network is preferable in the case of a large shock since interconnections serve as a mechanism for shock propagation. Many other theoretical or simulation-based works have studied the relevance of the various possible channels of contagion in the propagation of shocks and the implications for financial stability ${ }^{1}$. The work in this area usually corroborates with the "robust-yet-fragile" tendency of financial systems described by Gai and Kapadia (2010) in which the probability of contagion may be low but, when problems occur, the effects can be widespread

The statistical challenge in analyzing network data is to describe the potential dependence among nodes. For instance, in social network data the probability of a tie between two individuals increases as the characteristics of them become more similar (homophily). Equally important is the individual level heterogeneity, reflected in differences in observed characteristics, which plays an important role in the creation of new links. However, a main concern is the unobserved heterogeneity (see, e.g., Fafchamps et al. 2010).

In this paper, we present a model to handle dynamic network data with directed

\footnotetext{
${ }^{1}$ See Hüser (2015) and Glasserman and Young (2016) for recent reviews of the extensive literature on channels of contagion in financial networks.
} 
binary edges based on the latent space approach ${ }^{2}$. The model was originally introduced by Hoff et al. (2002) for static networks and later extended by Krivitsky et al. (2009) to account for clustering and homophily which is often observed in network data. Then, Sarkar and Moore (2005), Durante and Dunson (2014) and Sewell and Chen (2015) have proposed latent space models for dynamic networks.

In this model, the latent space refers to a space of unobserved characteristics that affect link formation. The model assumes that the presence of a link between two banks is independent of all other links in the system, given the unobserved positions in the latent space and observed characteristics of the nodes. Each node has a position in this low dimensional Euclidean space and the closer two nodes are, the more likely they will form a link. Although the interdependence between dyads is not explicitly parameterized, in the latent space model they are represented by latent variables. For this reason, the model can account for higher-order dependencies such as reciprocity and transitivity which are usually present in network data. So, the observation of $i \rightarrow j$ and $j \rightarrow k$ suggests that the nodes $i, j$ and $k$ are not too far apart making the existence of ties $j \rightarrow i$ (reciprocity) and $i \rightarrow k$ (transitivity) more probable. Estimation of the latent positions and the parameters of the model is done within a Bayesian framework.

A contribution of the paper is to use the dynamic latent space approach of Sewell and Chen (2015) to model monthly networks of directed interbank linkages. The latent space model has been mostly used for modeling social networks but it has not been applied to the study of financial networks so far. Although financial exposures are naturally represented by a weighted network, the choice of using a directed network representation is motivated by our aim to evaluate the ability of the model to characterize the basic structure of the network, which is the presence or absence of a link.

In this dynamic model each node has a temporal trajectory in the latent space. Hence, we expect to have a better understanding of how the network evolves over time and the behavior of individual banks instead of conducting separate analyses for each network or averaging graph statistics. We extended the model to include in the observation equation covariates that measures characteristics of the pair of banks and we are able to investigate how these pair-specific measures as size, liquidity, etc., affect the probability of forming a lending connection.

\footnotetext{
${ }^{2}$ The "latent space" has been used in economics to account for the unobserved homophily that might affect the formation and behavior of social networks (Goldsmith-Pinkham and Imbens, 2013 and Graham, 2014). Iijima and Kamada (2016) develop a network formation model in which the benefit and the cost of link formation depend on the social distance between agents.
} 
The motivation to use a dynamic network model is that papers usually consider the network structure of banks' exposures as fixed. They do not consider strategic actions that other banks may take to reduce the exposure to a bank in distress or liquidity hoarding in moments of increased uncertainty about the health of the financial system. For example, Afonso et al. (2011) examine the impact of the financial crisis of 2008 on the US interbank market and show how banks have become more restrictive in their lending operations after the Lehman Brothers' bankruptcy. Squartini et al. (2013) show the changes in the topology of the Dutch interbank network over the period 1998-2008. Despite the importance of contributions, a limited number of empirical papers explore the dynamics of financial networks. Most of them measure certain graph statistics over time instead of modeling the network dynamics in order to understand the changes in the network topology (Giraitis et al. 2016) The reason for this is the greater complexity in modeling dynamic network data given the dependence structures of networks observed over discrete time intervals. Further motivation is that currently central bank authorities collect a huge amount of data on bilateral exposures of financial institutions or they are able to derive them from payment system records 4 . Using the increasing availability of longitudinal data, researchers can model how the network evolves over time to make inference on its dependence structure.

We apply this methodology to analyze two different datasets: the unsecured and the secured interbank lending networks. We analyze these two networks separately since banks face distinct risks when lending in these markets. In unsecured lending, loans are not collateralised so lenders are directly exposed to loss in the case of borrowers default. In secured lending, which in our case is represented by repurchase agreements (repo) collateralized with government bonds, the loss is limited by the collateral value. In the former, counterparty risk plays a key role (see, e.g., Afonso et al., 2011) while in the latter the quality of collateral is an issue in moments of distress (see, e.g., Krishnamurthy et al., 2014). Despite the differences in risk assessment, graph statistics of the two networks are quite similar.

We estimate the model with the latent space but an important question is whether it improves the model's goodness-of-fit. The latent space is expected to account for the unobserved bank attributes that affect the link formation and improve the model's fit.

\footnotetext{
${ }^{3}$ For instance, Craig and von Peter (2014) track the number and composition of banks in the core and periphery on a quarterly basis as an evidence of tiering in the German banking system. They use the blockmodeling approach.

${ }^{4}$ The algorithms to extract the information from large value payment systems are usually based on the work of Furfine (1999) and later refinements.
} 
Hence, we run a model in which the probability of a tie depends only on observed covariates $\mathbf{x}_{i j t}$ and a second one in which the latent space is included. The former model is simply a logistic regression model in which directed ties are the dependent variables. We assess the in-sample and the out-of-sample link predictions of the two models. In addition, we evaluate the adequacy of them by comparing some selected graph statistics calculated for the observed data with their distributions obtained from a large number of networks simulated according to the fitted model (see, e.g., Hunter et al., 2008; Durante et al., 2017). If the simulated network does not resemble the observed network for a particular statistic, it is an indication of the model's lack of fit. We use this procedure to assess the model's fit considering a set of graph statistics which are believed to represent important structural properties of interbank networks such as degree distribution, reciprocity and assortativity.

Our main results show that the model which incorporates observed bank's characteristics and a latent space to account for the unobserved ones is able to capture some features of the dyadic data such as transitivity. We show that the inclusion of a low dimensional latent space is essential to account for dependencies that exist among nodes. Further, the distribution of graph statistics for the simulated networks based on the model with a latent space are much closer to the observed values when compared to the model without a latent space. On the other hand, the model in which the probability of forming a tie depends only on observed characteristics of banks has a poor fit. Link predictions are much worse and the distribution of most graph statistics for the simulated networks are far from the observed values. Therefore, models that do not incorporate dependencies in the analysis of network data will suffer from misspecification which may lead to invalid conclusions.

Lastly, the latent space approach is related to other statistical models for the analysis of network data. The methodology for dynamic network analysis is less developed since most of the models are for modeling static network 5 . However, given the importance of the subject, statistical models for the analysis of longitudinal network data are emerging. Examples of dynamic network models are the stochastic actor-based model (Snijders and Steglich, 2015) and the extension of the Exponential Random Graph Model (ERGM) for modeling dynamic networks (Hanneke et al., 2010). The former assumes that the objective function depends on preferences for certain types of social structures, such as reciprocated dyads or transitive triads while the latter depends

\footnotetext{
${ }^{5}$ Some models are intended for static networks but the processes for link creation or modification are dynamic. Examples are the Erdos-Renyi random graphs, preferential attachment and small-world models. See Goldenberg et al. (2009) for a survey of static and dynamic network models.
} 
on counts of specific network structures such as edges, triangles, k-stars, etc. Other methodology related to the latent space approach is the mixed membership stochastic blockmodel (Xing et al., 2010) which allows each node to belong to multiple blocks with fractional membership. In general, the models assume a continuous or a discrete Markov process to represent the network dynamics. However, the first two modeling approaches assume a homogeneous representation of the network behavior while the last ones allow for nodal heterogeneity in model parameters. Finally, a different approach combines agent-based modeling and the literature on strategic network formation to gain some economic intuition on the network formation process. Banks' utility functions are defined at the start and network formation follows a game theoretical approach (see e.g. Blasques et al., 2015).

The rest of the paper is organized as follows. Section 2 describes the latent space model for dynamic networks. Section 3 provides an explanation of the Bayesian estimation of model parameters. Section 4 describes the data on unsecured interbank exposures, repo transactions and bank variables. In Section 5 we analyze the two dynamic networks and report the main results and in Section 6 we discuss the importance of the latent space. Finally, in Section 7 we present a brief conclusion.

\section{The model}

In this section we describe the dynamic latent space approach (Sewell and Chen, 2015) for modeling the interbank network. The borrowing and lending relationships of the interbank market can be represented by a graph $\mathcal{G}_{t}=\left(\mathcal{N}, \mathcal{E}_{t}\right)$ where $\mathcal{N}$ is the set of all nodes and $\mathcal{E}_{t}$ is the set of edges at time $t \in \mathcal{T}=\{1,2, \ldots, T\}$. The number of nodes which represent financial institutions is $n=|\mathcal{N}|$. It will be assumed that $\mathcal{E}_{t}$ consists of directed edges representing whether or not a lending or a borrowing relationship exists between two banks at time $t$.

Each node $i$ has an unobserved position in a $p$-dimensional Euclidean latent space $\mathbb{R}^{p}$. Let $\mathbf{z}_{i t}$ represent the $p$-dimensional vector of the $i^{\text {th }}$ node's latent position at time $t$ and $\mathbf{Z}_{t}$ is the $n \times p$ matrix whose $i^{t h}$ row is $\mathbf{z}_{i t}$. The $n \times n$ square matrix $\mathbf{Y}_{t}=\left\{y_{i j t}\right\}$ is the adjacency matrix of the directed network observed at time $t$ where $y_{i j t}=1$ if bank $i$ has a credit exposure to bank $j$ at time $t$ and 0 otherwise. There are also a vector $\mathbf{x}_{i j t}$ and a $n \times n \times d$ array $\mathbf{X}_{t}$ of observed characteristics of the dyads.

It is assumed that the latent node positions follow a Markov process. It is also assumed that the observed graph at time $t$ is conditionally independent of all other 


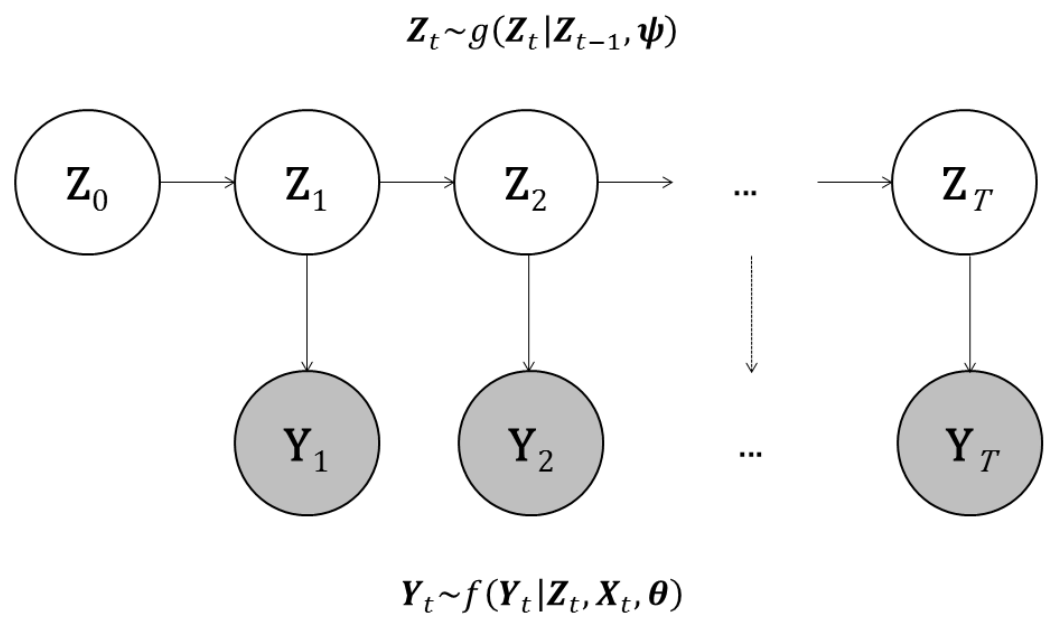

Figure 1: Graphical representation the Markovian dependencies between states $\mathbf{Z}_{t}$ and observations $\mathbf{Y}_{t}$.

graphs, given the latent positions and dyadic-level covariate information at time $t$. These assumptions lead to the representation of the temporal series of graphs as a state space model given in terms of densities by:

$$
\begin{aligned}
\mathbf{Y}_{t} & \sim f\left(\mathbf{Y}_{t} \mid \mathbf{Z}_{t}, \mathbf{X}_{t}, \boldsymbol{\beta}\right) \\
\mathbf{Z}_{t} & \sim g\left(\mathbf{Z}_{t} \mid \mathbf{Z}_{t-1}, \boldsymbol{\psi}\right)
\end{aligned}
$$

for $t=1,2, \ldots, T$, where $\boldsymbol{\psi}$ and $\boldsymbol{\beta}$ are sets of model parameters. Equation (1) is the observation equation that relates the observed graphs $\mathbf{Y}_{t}$ to the latent state variable $\mathbf{Z}_{t}$ representing the positions of nodes in a low dimensional Euclidean space. Equation (22) is the state transition equation that governs the time evolution of the unobserved latent state. A graphical representation of the dependencies between different states and observations is shown in Figure 1.

Xing et al. (2010) and Sewell and Chen (2015) assume that the evolution of the latent node positions follows a random walk. They show that even this simple model provides a better representation of the data than a static model that ignores time dependence between networks. Therefore, the initial distribution of the latent node 
positions and transition equation for $t=2,3, \ldots, T$ are given by

$$
\begin{gathered}
\pi\left(\mathbf{Z}_{1} \mid \boldsymbol{\psi}\right)=\prod_{i=1}^{n} \operatorname{MVN}_{p}\left(\mathbf{z}_{i 1} \mid \mathbf{0}, \tau^{2} \mathbf{I}_{p}\right) \\
\pi\left(\mathbf{Z}_{t} \mid \mathbf{Z}_{t-1}, \boldsymbol{\psi}\right)=\prod_{i=1}^{n} \operatorname{MVN}_{p}\left(\mathbf{z}_{i t} \mid \mathbf{z}_{i(t-1)}, \sigma^{2} \mathbf{I}_{p}\right),
\end{gathered}
$$

where $\mathbf{I}_{p}$ is the $p \times p$ identity matrix, $\operatorname{MVN}_{p}(\mathbf{z} \mid \boldsymbol{\mu}, \boldsymbol{\Sigma})$ denotes the $p$-dimensional multivariate normal probability density function with mean $\boldsymbol{\mu}$ and covariance matrix $\boldsymbol{\Sigma}$ evaluated at $\mathbf{z}$.

The logistic regression model is a convenient parameterization for the link probability (Hoff et al. 2002). The authors take a conditional independence approach to modeling by assuming that the probability of a link between two nodes is conditionally independent of all other links, given the latent positions and the observed characteristics of the dyad:

$$
\mathbb{P}\left(y_{i j t}=1 \mid \mathbf{z}_{i t}, \mathbf{z}_{j t}, \mathbf{x}_{i j t}, \boldsymbol{\beta}\right)=\frac{\exp \left(y_{i j t} \eta_{i j t}\right)}{1+\exp \left(\eta_{i j t}\right)} .
$$

In general, the logit ${ }^{6}$ of the link probability in latent space models is written as a linear function of covariates and a function of the distance of latent positions. Using the original parameterization of Hoff et al. (2002) for the dynamic network case, we have:

$$
\eta_{i j t}=\operatorname{logit}\left(\mathbb{P}\left(y_{i j t}=1 \mid \cdot\right)\right)=\beta_{0}+\boldsymbol{\beta}^{\prime} \mathbf{x}_{i j t}-d_{i j t}
$$

where the constant controls the overall density of the network, $\boldsymbol{\beta}$ is a vector of unknown parameters, $\mathbf{x}_{i j t}$ is a $d$-length vector of dyad specific covariates and $d_{i j t}=\left\|\mathbf{z}_{i t}-\mathbf{z}_{j t}\right\|$ is the Euclidean distance between nodes $i$ and $j$ within the latent space at time $t$. Hoff et al. (2002) call this formulation the distance model.

The distance model (equation 6) has a simple interpretation. The likelihood of a tie between $i$ and $j$ is a function of banks' observed and unobserved characteristics that might affect network formation. Examples of observed bank characteristics can be asset size, deposits or available liquidity. Unobserved latent characteristics can be for example private information about counterparty risk or the existence of a relationship between two banks in other markets which affects the probability of a link in the observed network. In this setting, the latent space can be interpreted as a characteristic space where banks that are closer together have a higher probability of forming lending or

\footnotetext{
${ }^{6} \operatorname{logit}(p)=\log (p /(1-p))$.
} 
borrowing relationships. Banks' positions in this space can help identify groups of banks that are similar to each other in terms of lending and borrowing behavior, after controlling for the explanatory variables.

In equation 6 we included only observed variables of the pair of banks and their positions in latent space to account for the unobserved characteristics that affect link formation. As we show in Sections 4 and 5 the links between banks are quite stable over the period of analysis, and macroeconomic variables such as GDP do not seem to affect the link formation process. One explanation could be the different frequency of observation of the variables. The networks are based on monthly data that are endof-month exposures or aggregation of daily data; to capture the effect of low frequency data on the formation of links we may need to analyze longer periods.

Finally, the likelihood of the conditional independence model is:

$$
\mathbb{P}\left(\mathbf{Y}_{1: T} \mid \mathbf{Z}_{1: T}, \mathbf{X}_{1: T}, \boldsymbol{\beta}\right)=\prod_{t=1}^{T} \prod_{i \neq j} \mathbb{P}\left(y_{i j t} \mid \mathbf{z}_{i t}, \mathbf{z}_{j t}, \mathbf{x}_{i j t}, \boldsymbol{\beta}\right)=\prod_{t=1}^{T} \prod_{i \neq j} \frac{\exp \left(y_{i j t} \eta_{i j t}\right)}{1+\exp \left(\eta_{i j t}\right)} .
$$

\section{Estimation}

A Bayesian approach is used to obtain estimates of the latent space positions and of the unknown parameters simultaneously. The posterior distribution

$$
\pi\left(\mathbf{Z}_{1: T}, \boldsymbol{\psi}, \boldsymbol{\beta} \mid \mathbf{Y}_{1: T}, \mathbf{X}_{1: T}\right)
$$

where $\boldsymbol{\psi}=\left(\tau^{2}, \sigma^{2}\right)$, is sampled using the Metropolis-Hastings algorithm within Gibbs sampling.

One problem of the model is that the likelihood is a function of the latent positions only through their distances. However, the distance is invariant under reflection, rotation and translation of the latent positions and, consequently, for each $n \times p$ matrix of latent positions $\mathbf{Z}_{t}$ there is an infinite number of other positions given the same $\log$-likelihood, i.e., $\log \mathbb{P}\left(\mathbf{Y}_{t} \mid \mathbf{Z}_{t}, \ldots\right)=\log \mathbb{P}\left(\mathbf{Y}_{t} \mid \mathbf{Z}_{t}^{*}, \ldots\right)$ for any $\mathbf{Z}_{t}^{*}$ that is equivalent to $\mathbf{Z}_{t}$ under the operations of reflection, rotation and translation.

To resolve the problem of the non-uniqueness of the estimates, Hoff et al. (2002) perform a Procrustean transformation on $\mathbf{Z}_{t}$. The goal is to transform a given matrix to be as close as possible to the target matrix by any combination of reflection, rotation 
and translation 7 . First, we choose the initial latent positions to be the $(n d) \times T$ target matrix $\mathbf{Z}_{0}$. Then, after each iteration of the MCMC algorithm the coordinates of $\widetilde{\mathbf{Z}}=$ $\left(\mathbf{Z}_{1}^{\prime}, \ldots, \mathbf{Z}_{T}^{\prime}\right)^{\prime}$ are transformed to be as close as possible to the corresponding coordinates of $\mathbf{Z}_{0}$. The transformation does not change the distances nor the likelihood but it approximately fixes the location of $\widetilde{\mathbf{Z}}$ and makes MCMC iterations comparable.

We use standard prior distributions and the values of the prior hyperparameters are set to make the priors weakly informative. The prior distributions for the parameters are set as follows: $\tau^{2} \sim \operatorname{IG}\left(\nu_{\tau} / 2, \nu_{\tau} \xi_{\tau}^{2} / 2\right), \sigma^{2} \sim \operatorname{IG}\left(\nu_{\sigma} / 2, \nu_{\sigma} \xi_{\sigma}^{2} / 2\right)$ and $\boldsymbol{\beta} \sim \operatorname{MVN}\left(\boldsymbol{\mu}_{\beta}, \boldsymbol{\Sigma}_{\beta}\right)$, where IG and MVN are the inverse gamma and the multivariate normal distributions, respectively. The inverse gamma distribution was chosen as it is a conjugate prior for the unknown parameters $\tau^{2}$ and $\sigma^{2}$.

The values of prior hyperparameters $\left(\nu_{\tau}, \xi_{\tau}^{2}, \nu_{\sigma}, \xi_{\sigma}^{2}, \boldsymbol{\mu}_{\beta}, \boldsymbol{\Sigma}_{\beta}\right)$ are set based on the suggestions of Sewell and Chen (2015). In general, we use relatively diffuse prior distributions. The shape and scale parameters for $\tau^{2}$ were set to low values in order to have a weakly informative prior. The same procedure was used for $\sigma^{2}$. In addition, the value of $\xi_{\tau}^{2}$ was set to be equal to the initial estimate of $\tau^{2}$ (equation 19). The prior for $\boldsymbol{\beta}$ has zero mean and large variance. Nonetheless, we found that the estimation is robust to changes in the values of prior hyperparameters and the convergence of the parameters is not affect.

\subsection{Posterior sampling and the MCMC algorithm}

We implement a Markov Chain Monte Carlo (MCMC) algorithm to sample from the posterior to obtain estimates of the latent positions and other unknown parameters of the model. The model parameters $\tau^{2}$ and $\sigma^{2}$ are sampled directly from their full conditional distributions using the Gibbs sampler while conditional distributions which do not have closed forms are sampled using a standard Metropolis-Hastings (MH) algorithm. The conditional distributions used in the MH steps are given in Appendix A where we derive in detail the conditional distribution of $\mathbf{Z}_{t}$ as well as the conditional distributions of the latent position $\mathbf{z}_{i t}$ and parameters $\left(\tau^{2}, \sigma^{2}, \boldsymbol{\beta}\right)$.

In order to reduce the time for the Markov chain to get to stationarity, we seek reasonable estimates for the initial values of the latent positions $\mathbf{Z}_{1: T}$ and the parameters

${ }^{7}$ Formally, the Procrustean transformation of $\widetilde{\mathbf{Z}}$ using $\mathbf{Z}_{0}$ as the target matrix solves the problem:

$$
\operatorname{argmin}_{T \widetilde{\mathbf{Z}}} \operatorname{tr}\left(\mathbf{Z}_{0}-T \widetilde{\mathbf{Z}}\right)^{\prime}\left(\mathbf{Z}_{0}-T \widetilde{\mathbf{Z}}\right)
$$

where $T$ is a set of reflections, rotations and translations. 
$\tau^{2}, \sigma^{2}$, and $\boldsymbol{\beta}$. The procedure to obtain the starting values is detailed in Appendix B. Given the starting values of $\mathbf{Z}_{1: T}, \tau^{2}, \sigma^{2}$ and $\boldsymbol{\beta}$ and current values at scan $s$ of the Markov chain, the MH algorithm generates a new set of parameter values as follows:

1. For $t=1, \ldots, T$ and for $i=1, \ldots, n$, sample $\mathbf{z}_{i t}^{(s+1)}$ using a MH step with a normal random walk proposal distribution.

2. Sample $\boldsymbol{\beta}^{(s+1)}$ using a MH step with a multivariate normal random walk proposal distribution.

3. Sample $\tau^{2(s+1)}$ from its full conditional distribution.

4. Sample $\sigma^{2(s+1)}$ from its full conditional distribution.

Finally, the variance of the proposal distributions are tuned automatically to achieve acceptance rates of $35 \%$ based on the algorithm proposed by Haario et al. (2005).

\section{Data}

The Central Bank of Brazil collects and processes data of exposures between financial institutions from different sources as credit register, securities custody and settlement systems and shares and derivatives central counterparties. The dataset built by the central bank includes exposures of banks, credit unions, securities dealers and other financial institutions.

We restrict our analyses to exposures between banks. Using this sample the number of financial institutions decrease from more than 1,500 to approximately 135 without losing relevance, since financial institutions other than banks account for only $2.7 \%$ of the financial system's total assets as of December 2014. Besides, the Brazilian financial system is highly concentrated. Among more than one hundred domestic and foreign banks, six large institutions account for $78.4 \%$ of the financial system's total assets. As a consequence of banking concentration, interfinancial assets of banks account for $98.0 \%$ of the interfinancial asset of the financial system. It is worth noting that exposures are measured on a consolidated basis thus the term bank refers indistinctly to an individual bank or to a banking group.

In our study we analyze the networks of unsecured interbank exposures and repo transactions from July 2012 to June 2013 (12 months). The unsecured interbank data 
are available on a monthly basis and consist of short and long term exposures between banks. Exposures are measured as end-of-month gross values. There is no netting between exposures of banks $i$ and $j$. These exposures arise mainly from interbank deposits, credit, loan purchase agreements and interbank onlending. A smaller fraction of exposures are due to certificates of deposit, OTC derivatives such as swaps, and holdings of securities issued by other financial institutions.

The data on repurchase agreements (repo) with government bonds as collateral is from Selic, the system for settlement and custody of government bonds managed by the Central Bank of Brazil. Excluding transactions with the central bank, overnight repos account for almost all operations between financial institutions. There is no netting between exposures of banks $i$ and $j$ or between the amount lent and the collateral value. Data are available on a daily basis but we have chosen to analyze monthly aggregated networks. To do so, for each month we summed the daily exposures of each pair of banks. The data aggregation is necessary since the MCMC algorithm cannot handle daily network data due to the computational burden associated with the estimation of so many parameters. Besides, aggregated networks are expect to provide a better view of the interbank market structures as the randomness of daily networks may result in misleading inferences (Finger et al., 2013).

Figure 2 shows the number of links between banks and the traded volumes 9 in the unsecured and the repo interbank markets. We can see that traded volumes and number of links for both networks have increased over the year. The difference in volumes traded in unsecured and secured markets is explained by the fact that most of repo transactions have the central bank as counterparty while repos between banks account for a small fraction of the market.

\subsection{The topology of the Brazilian interbank networks}

Some characteristics of the Brazilian interbank network are analyzed in other works (Cajueiro and Tabak, 2008; Cont et al., 2013; Tabak et al., 2014; Silva et al., 2015). The papers differ in the financial instruments used to build the networks and the sample time span. Even so, they have found that the Brazilian interbank networks are quite similar to networks from other countries. For instance, the networks are sparse and show a core periphery structure while the degree distribution seems to follow a power

\footnotetext{
${ }^{8}$ Onlending refers to loans of borrowed funds from other banks. In our database large onlending exposures arise mainly when banks lend money borrowed from the Brazilian development bank.

${ }^{9}$ The volumes traded were deflated by the Brazilian consumer price index IPCA.
} 

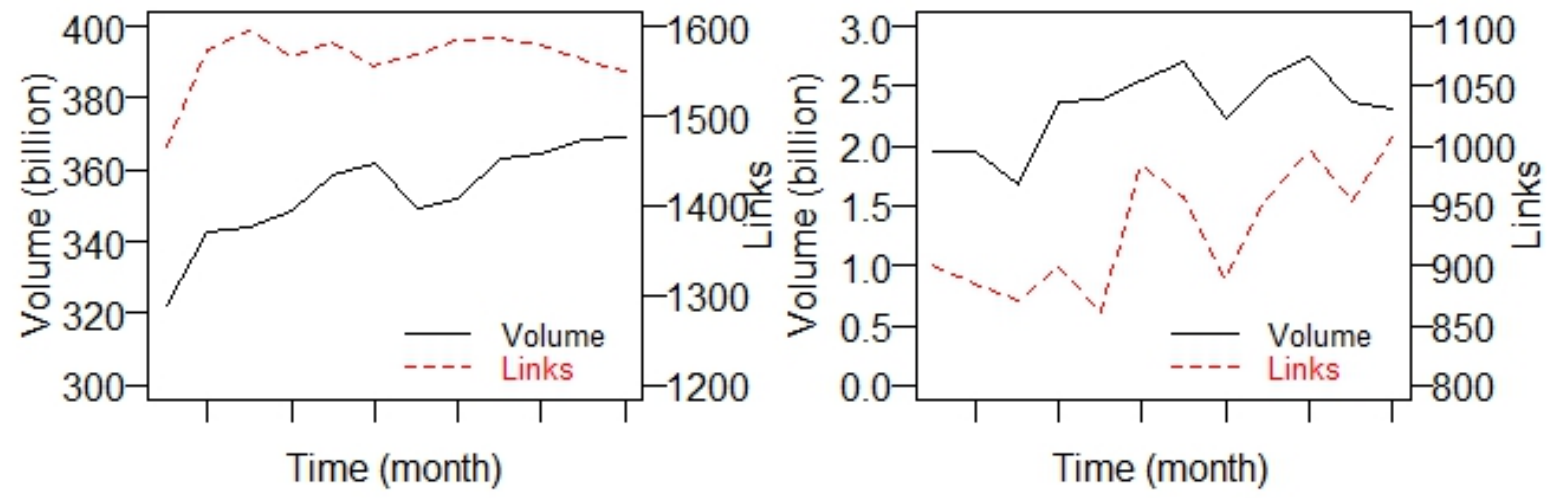

Figure 2: Traded volume (black solid line) and number of links between banks (red dashed line) from July, 2012 to June, 2013.

law.

Table 1 presents the evolution of some network statistics of the unsecured and the repo interbank networks. In Appendix C we provide an explanation of the network statistics used in the table. Our sample is from July 2012 to June 2013 but since the variation between months is low, we present the results for only six different months.

The in-degree of a node $i$ is the number of edges terminating at it and out-degree is the number of edges originating in node $i$. In our interbank network they represent respectively the number of creditors and debtors of a bank. It is possible to see by the coefficient of variation (standard deviation of degree/mean degree) that the out-degree distribution of the unsecured network is more dispersed than the in-degree distribution while for the repo network it is the contrary. The maximum in/out-degrees of the unsecured network are 68 and 107, respectively, and 82 and 42 for the repo network.

The density of a network is the ratio of the number of existing edges to the number of possible edges. Both networks are sparse as the mean density is 0.10 or lower. Transitivity or clustering coefficient is the probability that adjacent nodes of a node are also connected. Reciprocity is the proportion of the total number of reciprocated edges to the total number of edges. Transitivity and reciprocity are high in both networks and almost constant.

The average path length is the average length of the shortest paths between nodes while the diameter is the length of the longest path in a graph. It is possible to see that the unsecured network exhibits smaller distances between nodes. Assortativity is the correlation of degrees in adjacent nodes. The degree correlations are negative in both 
Table 1: Summary of graph statistics for the unsecured and the repo interbank networks.

\begin{tabular}{|c|c|c|c|c|c|c|c|}
\hline & Jul 2012 & Sep 2012 & Nov 2012 & Jan 2013 & Mar 2013 & May 2013 & Mean \\
\hline \multicolumn{8}{|l|}{ Unsecured network } \\
\hline In-degree Mean & 12.23 & 13.18 & 12.96 & 12.76 & 12.90 & 12.81 & 12.81 \\
\hline In-degree Coef. var. & 0.95 & 0.94 & 0.94 & 0.95 & 0.96 & 0.94 & 0.95 \\
\hline Out-degree Mean & 12.23 & 13.18 & 12.96 & 12.76 & 12.90 & 12.81 & 12.81 \\
\hline Out-degree Coef. var. & 1.47 & 1.41 & 1.44 & 1.45 & 1.44 & 1.44 & 1.44 \\
\hline Density & 0.10 & 0.11 & 0.11 & 0.10 & 0.11 & 0.11 & 0.11 \\
\hline Transitivity & 0.35 & 0.37 & 0.36 & 0.37 & 0.36 & 0.36 & 0.36 \\
\hline Reciprocity & 0.50 & 0.53 & 0.51 & 0.51 & 0.52 & 0.51 & 0.51 \\
\hline Average path length & 2.08 & 2.08 & 2.07 & 2.09 & 2.06 & 2.08 & 2.08 \\
\hline Diameter & 4 & 4 & 4 & 4 & 4 & 4 & 4 \\
\hline Assortativity & -0.37 & -0.37 & -0.37 & -0.37 & -0.37 & -0.36 & -0.37 \\
\hline \multicolumn{8}{|l|}{ Repo network } \\
\hline In-degree Mean & 7.96 & 7.71 & 7.61 & 8.39 & 8.38 & 8.36 & 8.07 \\
\hline In-degree Coef. var. & 1.62 & 1.58 & 1.51 & 1.56 & 1.61 & 1.48 & 1.56 \\
\hline Out-degree Mean & 7.96 & 7.71 & 7.61 & 8.39 & 8.38 & 8.36 & 8.07 \\
\hline Out-degree Coef. var. & 0.82 & 0.85 & 0.87 & 0.85 & 0.76 & 0.82 & 0.83 \\
\hline Density & 0.07 & 0.07 & 0.07 & 0.07 & 0.07 & 0.07 & 0.07 \\
\hline Transitivity & 0.31 & 0.31 & 0.32 & 0.34 & 0.31 & 0.31 & 0.32 \\
\hline Reciprocity & 0.48 & 0.49 & 0.45 & 0.46 & 0.45 & 0.50 & 0.47 \\
\hline Average path length & 2.32 & 2.34 & 2.35 & 2.24 & 2.26 & 2.25 & 2.29 \\
\hline Diameter & 5 & 5 & 5 & 5 & 5 & 5 & 5 \\
\hline Assortativity & -0.33 & -0.32 & -0.34 & -0.35 & -0.33 & -0.35 & -0.34 \\
\hline
\end{tabular}


networks, showing that low degree nodes have a higher tendency to be connected to high degree nodes. Finally, it can be seen from the tables that the networks are quite stable since network statistics show little variation over the period of analysis.

\subsection{Banks' observed characteristics}

We select a set of covariates that may have an effect on the link formation process. Some of these covariates were used by Cocco et al. (2009), Affinito (2012) and Afonso et al. (2014) to investigate the existence of lending relationships in the interbank market. As found by these papers we expect that some observable characteristics of banks which we use as similarity or dissimilarity measures or a complementarity measure on each pair of institutions will have an effect on the formation of ties.

We use monthly balance sheet information which are published by the Central Bank of Brazil ${ }^{10}$ to construct the covariates of bank characteristics. In particular, we include bank size defined as the natural logarithm $(\log )$ of total assets. Bank size is expected to explain much of the connections in the data since large institutions are usually in the core of the network acting as money center banks (Craig and von Peter. 2014). In addition, the analysis of the data shows that large banks have more connections than smaller banks and they lend more than they borrow from other institutions. We use credit to total assets and securities and derivatives to total assets as measures of asset structure which reflects banks' business models. We also include the ratio of non performing loans (loans that are overdue for more than 90 days) to outstanding loans and deposits to total assets. The former could be an indication of asset quality and the latter of funding stability.

Table 2 shows the descriptive statistics of the variables. The mean, standard deviation and minimum, maximum, $25 \%$ and $75 \%$ values were computed considering the whole sample. The heterogeneity in banks' characteristics is huge. For instance, the largest bank is 100,000 times bigger than the smallest one in terms of total assets. In the sample there are a few retail banks with a large base of depositors and many small banks which serve mainly small business.

As a measure of similarity or complementarity on observed characteristics of banks $i$ and $j$, we use the absolute value of the difference between the nodal covariates $x_{i t}$ and $x_{j t}$, i.e., the vector of covariates $\mathbf{x}_{i j t}$ is a function of $\mathbf{x}_{i j t}=\left|\mathbf{x}_{i t}-\mathbf{x}_{j t}\right|$. Hence, this model is symmetric, i.e., $\mathbb{P}\left(y_{i j t}=1 \mid \cdot\right)=\mathbb{P}\left(y_{j i t}=1 \mid \cdot\right)$, which we expect that it can account for the high reciprocity observed in the network data. We have tested other

\footnotetext{
${ }^{10}$ http://www4.bcb.gov.br/top50/port/top50.asp
} 
Table 2: Summary statistics of covariates from July, 2012 to June, 2013.

\begin{tabular}{|c|c|c|c|c|c|c|c|c|}
\hline Name & Definition & Obs. & Mean & St. dev. & Min & $25 \%$ & $75 \%$ & $\operatorname{Max}$ \\
\hline Size & $\log ($ total assets $)$ & 1476 & 14.675 & 2.371 & 9.871 & 12.903 & 16.114 & 21.407 \\
\hline Credit & $\begin{array}{l}\text { credit/total } \\
\text { assets }\end{array}$ & 1476 & 0.374 & 0.281 & 0 & 0.102 & 0.579 & 0.967 \\
\hline Securities & $\begin{array}{l}\text { securities and } \\
\text { derivatives/total } \\
\text { assets }\end{array}$ & 1476 & 0.198 & 0.213 & 0 & 0.057 & 0.249 & 0.969 \\
\hline$N P L$ & $\begin{array}{l}\text { non performing } \\
\text { loans/outstanding } \\
\text { loans }\end{array}$ & 1476 & 0.022 & 0.025 & 0 & 0.002 & 0.032 & 0.292 \\
\hline Deposits & $\begin{array}{l}\text { deposits/total as- } \\
\text { sets }\end{array}$ & 1476 & 0.326 & 0.230 & 0 & 0.119 & 0.502 & 0.831 \\
\hline
\end{tabular}

functional forms for the dyad covariates as the difference between nodal variables or the $\log \left(\mathbf{x}_{i t} / \mathbf{x}_{j t}\right)$, however these models do not fit the data well.

\section{Results}

\section{$5.1 \quad$ Unsecured network}

We analyze the Brazilian unsecured interbank market from July 2012 to June 2013. We have $T=12$ time periods and $n=123$ banks which accessed the market during this period. The networks are constructed based on end-of-month gross exposures which arise from bank $i$ claims on bank $j$. Thus, we have twelve $123 \times 123$ binary adjacency matrices where the edge $y_{i j t}$ of the matrix $\mathbf{Y}_{t}$ represents a exposure of bank $i$ to bank $j$ at time $t$.

We estimate the distance model (Eq. 6) and initialize the latent positions and parameters of the model as described in Appendix B. The initial values of $\tau_{0}^{2}$ and $\sigma_{0}^{2}$ were set to 0.1906 and 1 , respectively. Prior hyperparameters were set to $\nu_{\tau}=\nu_{\sigma}=2$, $\xi_{\tau}^{2}=\tau_{0}^{2}, \xi_{\sigma}^{2}=0.001$ and $\left(\boldsymbol{\mu}_{\beta}, \boldsymbol{\Sigma}_{\beta}\right)=(\mathbf{0}, 2 \cdot \mathbf{I})$.

One issue in the latent space approach is the selection of the dimension of latent variables $\mathbf{z}_{i t}$. There is not an automatic procedure for learning the dimension of the latent space but we would like to have a simple graphical representation of it. As visualization of the network is one possible motivation for using the latent space approach to modeling networks, $p$ is usually set to 2 but higher dimensions may be needed to rep- 
resent the network adequately. Hoff (2011) uses the Deviance Information Criterion 11 (Spiegelhalter et al., 2002), which can be computed from the output of the MCMC, to find the rank for reduced-rank decomposition of an array. Following the same procedure, we evaluate models having dimensions of $p=\{2,3,4\}$ and select the optimal dimension based on the computation of the DIC.

The model with 4 dimensions achieved the minimum DIC. We examined the fit of models having higher dimensions but the results had roughly the same performance at a cost of estimating $n$ more parameters per additional dimension.

Initially, we fit the model with $p=4$ without covariate information on the banks' characteristics. We run 100,000 iterations of the MCMC algorithm with a burn-in of 20,000 iterations. Visual inspection of the trace-plots shows that the length of the burn-in is sufficient for convergence. The Geweke (1992)'s convergence diagnostic for the equality of the means of the first and last part of the chain yielded Z-scores of -1.49, -1.26, 0.83 for $\tau^{2}, \sigma^{2}$ and $\beta_{0}$, which indicate convergence. Posterior values of model parameters $\tau^{2}, \sigma^{2}$ and $\beta_{0}$ are kept every 20 iterations to reduce autocorrelation of the samples. The MCMC trace plots, auto-correlation functions and marginal posterior distributions of $\tau^{2}, \sigma^{2}$ and $\beta_{0}$ are plotted in Figure 3 . The thinning of the Markov chain was able to reduce the autocorrelation of the $\tau^{2}$ and the $\beta_{0}$ samples. Although the auto-correlation of $\sigma^{2}$ is still high, we initialized the chain from different values and the results show that it converges.

Next, we investigate how bank characteristics as size, credit, deposits, etc., affect the probability of a pair of banks forming a connection. We estimate seven different specifications of the model including the following dyadic covariates: Size, Credit, Securities, NPL and Deposits. The fit of the model's different specifications is compared in three ways. First, we assess how well the model explains the data used to estimate the model. We obtain in-sample link predictions of $\mathbf{Y}_{1: T}$ using the posterior means of model's parameters and latent positions as inputs for the observation equation and then we compute the area under receiver operating characteristic curve (AUC). The ROC curve plots the true positive rate (sensitivity) as a function of the false positive rate (specificity); the closer AUC is to 1 the better is the fit, whereas random predictions imply an AUC of 0.5. In addition, we compute the AUC of the out-of-sample predictions of the model. To this end, we compute one step ahead predicted probabilities

\footnotetext{
${ }^{11}$ The DIC is intended as a generalization of Akaike's Information Criterion (AIC) for comparing complex models in which the number of free parameters is not clearly defined. It is defined as DIC $=$ $\bar{D}+p_{D}$, where $\bar{D}=-2 \mathbb{E}(\log p(y \mid \theta)), p_{D}=\bar{D}+2 \log p(y \mid \bar{\theta})$ is the effective number of parameters and $\bar{\theta}$ is the posterior mean of parameters.
} 

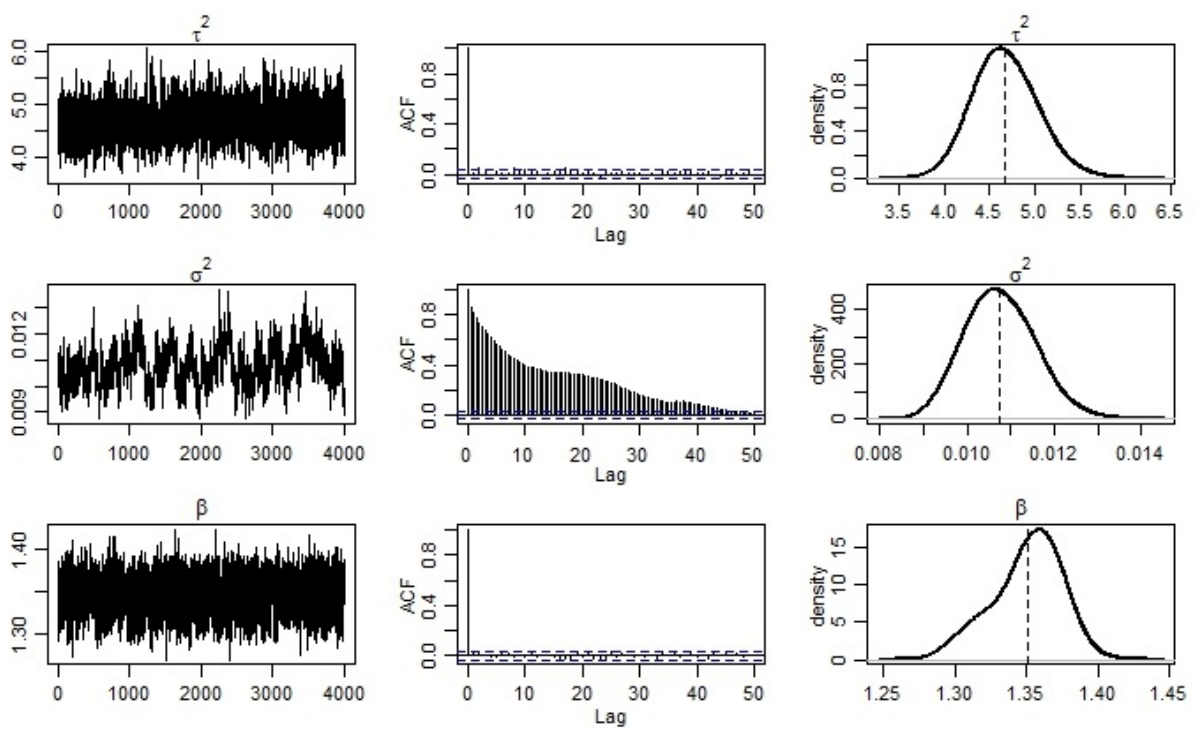

Figure 3: MCMC trace plots, auto-correlation functions and posterior distributions of $\tau^{2}, \sigma^{2}$ and $\beta_{0}$ for the unsecured network data. Vertical dashed lines show the mean of the posterior distributions of the parameters.

and compare the results to the observed network at time $T+1$. The out-of-sample predictions are based on the model estimate using networks observed from July 2012 to June 2013 and compared to the network observed at July 2013. Finally, the fit of each different specification is assessed based on the computation of the DIC.

In Table 3 we show the coefficients and AUC and DIC values for the model estimated including a different set of variables. The AUC values for the in-sample predictions show that with the exception of the models without covariates (AUC value of 0.8672 ) and without the covariate Size (AUC value of 0.8729 ) the models fit the data quite well. The AUC values are on average 0.92. The AUC values of the out-ofsample predictions are also high, showing that the model provides good one step ahead predicted probabilities as well. It is important to note that the improvement of the model's fit with the inclusion of other variables in addition to Size is not significant. The AUC values of the in-sample and out-of-sample predictions for specification 2 which includes only the variable Size and specification 6 which includes all variables are almost the same. When we compare the DIC values of the models we see that the model without covariates has the worst fit (the highest DIC value). The difference of the DIC values are not high, nonetheless the model with all covariates included excepting Deposits has the lowest DIC.

The estimate for the coefficient of Size in specification 2 is $0.82(0.02)$. The positive value implies that it is more probable for banks to have ties when the difference 
in their sizes is large compared to ties between banks of similar sizes. The coefficients of other variables (Securities, NPL and Deposits) are negative implying that there is a preference for ties between banks of similar characteristics. The coefficient of Credit is negative with the exception of the coefficient estimated in specification 6 although the highest posterior density interval is between zero. The inclusion of other variables does not improve the fit of the model significantly so we proceed our analyses based on the model that uses only covariate information on the size of the banks.

In order to allow the visualization of banks' positions we show in Figure 4 the posterior means of banks' latent positions for the first two dimensions. The figure shows the positions in two different dates: July 2012 (circles) and June 2013 (triangles). The six large institutions, which are considered domestic systemically important banks, are positioned near to each other and close to the center of the figure (green symbols), while small banks with few connections are on the border.

In Figure 5 we show boxplots of the distances each bank traveled in the latent space during the eleven month transitions. Sewell and Chen (2015) use the boxplots of the distance to evaluate the stability of the network. It can be seen that the moves of banks corresponding to each transition fall in a similar range implying that the dynamics of the network are nearly constant throughout the twelve months. Transitions to December 2012 and to January 2013 implied larger alterations in banks' positions, which can be explained by changes in the amount lent. The set outlier banks changes over the transitions but four small foreign banks are outliers in six or more months.

\subsection{Repo network}

We analyze the data on repo agreements collateralized with government bonds in this section. The daily data of overnight repo transactions from July 2012 to June 2013 are aggregated by months resulting in twelve networks $(T=12)$. We have the same sample of $n=123$ banks which accessed the market during this period resulting in a $123 \times 123$ binary adjacency matrix $\mathbf{Y}_{t}$. Each edge $y_{i j t}$ of $\mathbf{Y}_{t}$ represents the amount lent by bank $i$ to bank $j$ during month $t$.

We follow the same procedure of Section 5.1 to estimate the model. The latent positions and parameters of the model are initialized as described in Appendix B. The initial values of $\tau_{0}^{2}$ and $\sigma_{0}^{2}$ were set to 0.3552 and 1 , respectively. Prior hyperparameters were set to $\nu_{\tau}=\nu_{\sigma}=2, \xi_{\tau}^{2}=\tau_{0}^{2}, \xi_{\sigma}^{2}=0.001$ and $\left(\boldsymbol{\mu}_{\beta}, \boldsymbol{\Sigma}_{\beta}\right)=(\mathbf{0}, 2 \cdot \mathbf{I})$.

We evaluate models having dimensions of $p=\{2,3,4\}$ and the model with 4 dimensions achieved the minimum DIC. We run 100,000 iterations of the MCMC al- 


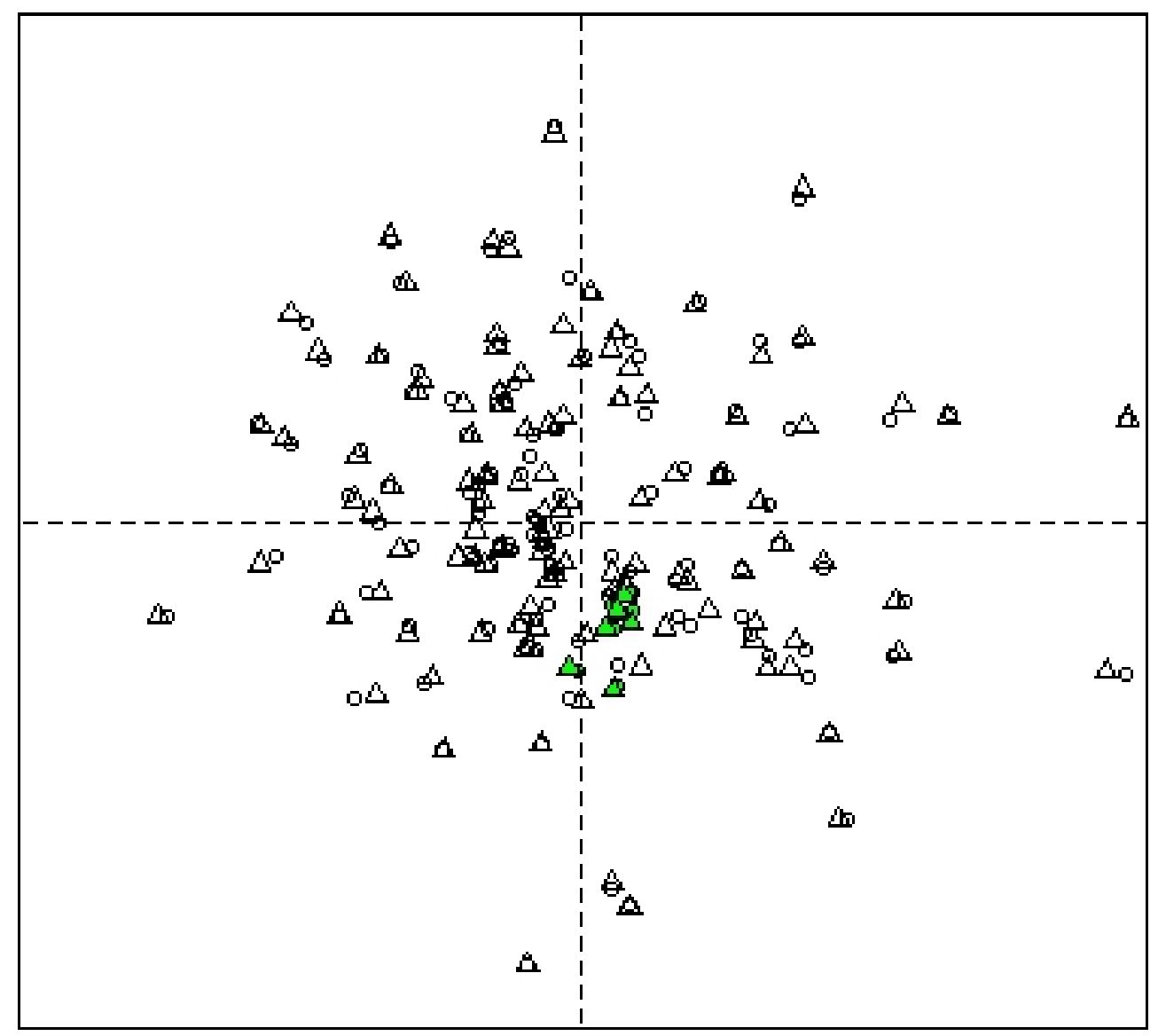

Figure 4: Posterior means of latent banks' positions for unsecured network data. Circles are positions in July, 2012 and triangles are the positions in June, 2013. Green symbols are the positions of the six largest banks. 


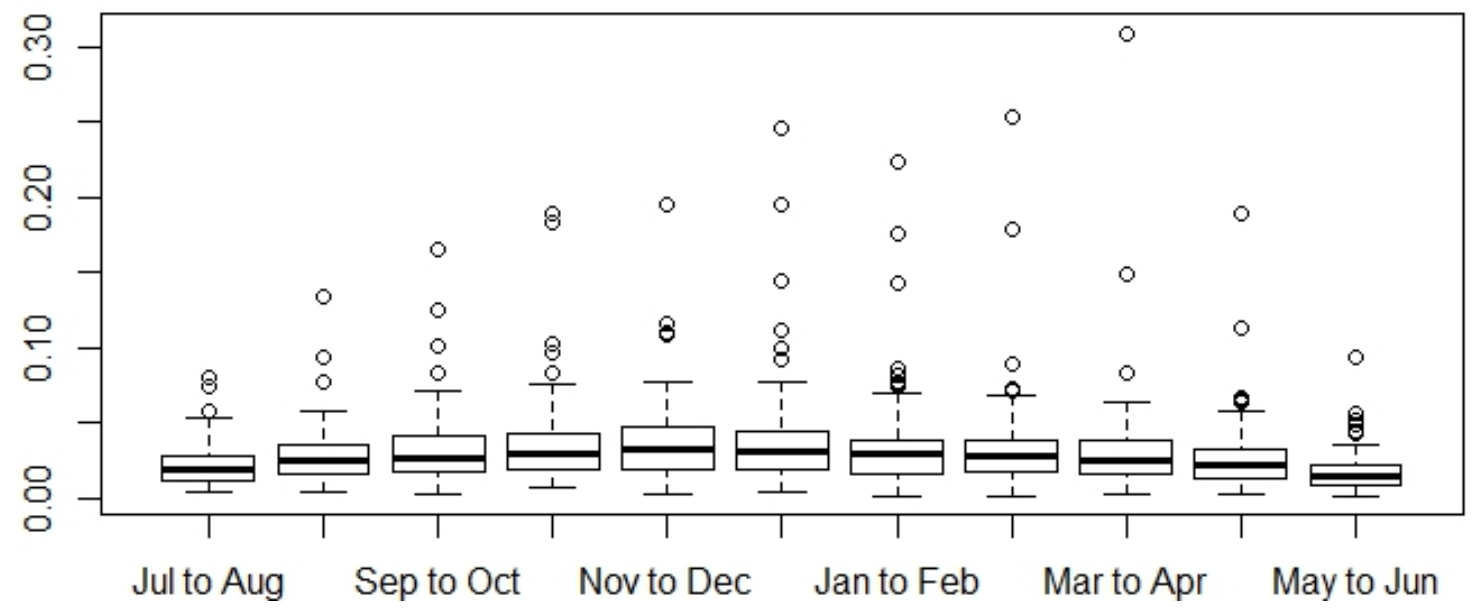

Figure 5: Boxplots of the distances traveled by banks in the latent space during twelve months for the unsecured network data.

gorithm with a burn-in of 20,000 iterations. The Geweke (1992)'s convergence test for $\tau^{2}, \sigma^{2}$ and $\beta_{0}$ indicated convergence (Z-scores of $0.02,-0.45,-0.46$, respectively). The MCMC trace plots of posterior values of $\tau^{2}, \sigma^{2}$ and $\beta_{0}$ after the burn-in and keeping values at every 20 iterations are given in Figure 6. The figure also shows the auto-correlation functions and marginal posterior distributions of the parameters. The thinning was able to reduce the autocorrelation of the samples of the Markov chain.

In order to investigate how bank characteristics affect the probability of a pair of banks forming a connection, we estimate seven different specifications of the model including the following dyadic covariates: Size, Credit, Securities, NPL and Deposits. We compare the AUC of the in-sample and out-of-sample predictions and the DIC of the model's different specifications. The out-of-sample predictions are based on the model estimated using networks observed from July 2012 to June 2013 and compared to the network observed at July, 2013. In Table 4 we show the coefficients and AUC and DIC values for the model estimated including different set of variables.

The AUC values for the in-sample predictions of all specifications are high, showing that the models fit the data quite well. The AUC values are approximately 0.94. The AUC values of the out-of-sample predictions are also high showing that the model provides good one step ahead predicted probabilities as well. The inclusion of the variable Size has the highest impact on AUC values whereas the model with all variables but Size has the lowest AUC. The model with only a constant has higher AUC values 

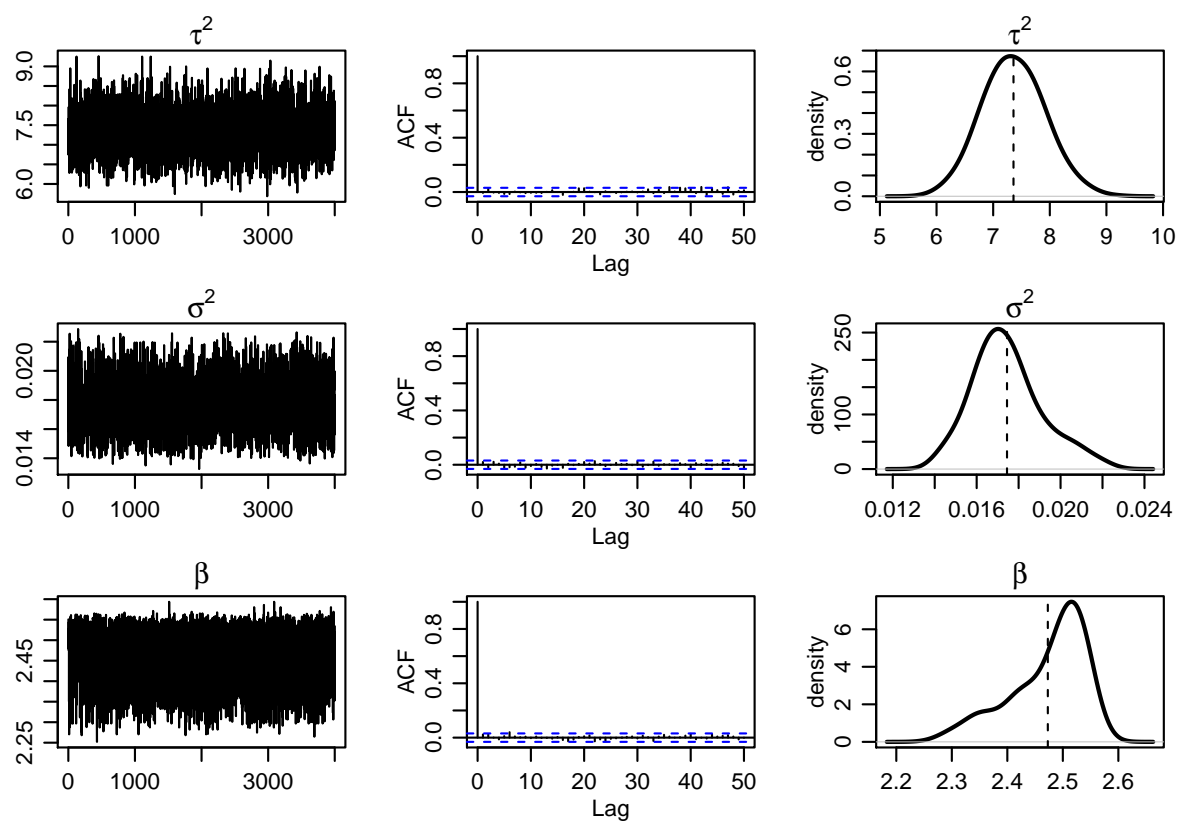

Figure 6: MCMC trace plots, auto-correlation functions and posterior distributions of $\tau^{2}, \sigma^{2}$ and $\beta_{0}$ for the repo network data. Vertical dashed lines show the mean of the posterior distributions of the parameters.

than this model. The model without covariates and the model with all variables with the exception of Size have the worst fit (the highest DIC values). The difference of the DIC values are not high, nonetheless the specification 3 has the lowest DIC.

Similar conclusions about the importance of Size are drawn for the repo network model. The improvement of the model's fit with the inclusion of other variables in addition to Size is not significant. Size coefficients are positive which imply that it is more probable for banks to have ties when the difference in their sizes is large compared to ties between banks of similar sizes. The coefficients of the other variables (Credit, Securities and Deposits) are negative implying that there is a preference for ties between banks of similar characteristics while the coefficients of NPL are positive.

\section{The latent space and model's goodness-of-fit}

We expect that the latent space can account for the unobserved bank attributes that affect the link formation and improve the model's fit. Hence, it is important to assess the fit of the model in which the probability of a tie depends only on observed covariates $\mathbf{x}_{i j t}$ and the model in which the latent space is included.

In Table 5 we present the coefficients of the model estimated without the latent 
space. It is a logistic regression model in which the dyads are independent to each other and dependencies among nodes in the network are not represented. Since the unsecured and the repo networks have similar characteristics, we present the results only for the model estimated using the unsecured network data.

The relations between banks are reciprocal and transitive. The average reciprocity and transitivity of the observed networks are 0.51 and 0.36 , respectively. However, the model without the latent space assumes that a link between a pair of banks is independent to other links. The estimated coefficients of both models have same signs with the exception of the constant that controls the overall density of the network. However, they are very different indicating that it is important to take the latent space into account. For instance, the coefficients of the model with a constant and variable Size are -2.95 and 0.25 for the model without the latent space while the coefficients for the model with the latent space are 0.57 and 0.82 .

The model's goodness of fit is evaluated computing the AUC of the in-sample and out-of-sample link predictions. To obtain the out-of-sample link predictions we estimate the model and compute the one step ahead predicted probabilities that are compared to the network observed in July 2013. The results show that the AUC of the in-sample and out-of-sample link predictions of the model without the latent space are on average just 0.70 compared to an AUC of 0.92 for the model with the latent space. Another fact is that the inclusion of other variables improved the fit of the model without the latent space.

In Figure 7 we show the ROC curve for the out-sample predictions of the models with and without the latent space and including only size of the banks as covariate. Although the predictions of the model with the latent space has a high AUC and performs better than the model without the latent space, it could not outperform the predictions of the simple method based on $\mathbf{Y}_{T}$ to predict $\mathbf{Y}_{T+1}$. Due to the persistence of the network links, this naive prediction method obtained higher true positive (sensitivity) and false positive $(1-$ specificity) rates than the latent space model.

Then, we evaluate model adequacy by comparing selected network statistics computed for the observed data with distributions induced by the statistical model (see, e.g., Durante et al., 2017). To perform this graphical test of goodness-of-fit we proceed as follows: first, we simulate a large number of networks from the estimated model. For each simulated network we compute a set of graph statistics of interest. Then, the distributions of graph statistics of the simulated data are compared to the values of the observed network. If they fall in the tails of the distributions, it is an indication of the 


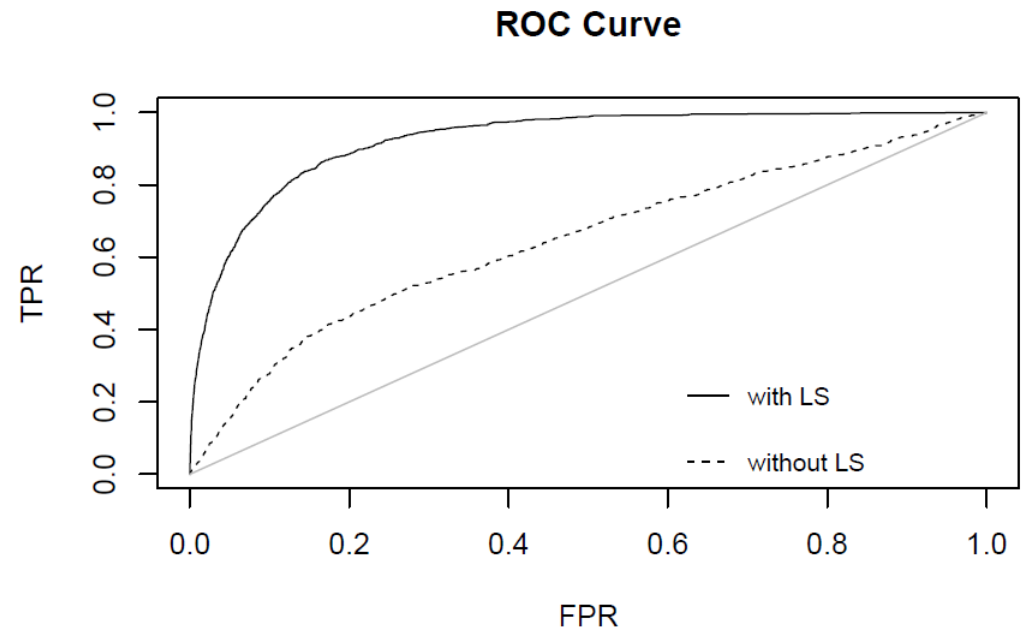

Figure 7: ROC curve showing the TPR (True Positive Rate) and the FPR (False Positive Rate) of the models with the latent space (solid line) and without the latent space (dashed line).

lack of fit.

In order to compare simulated networks and the observed one it is important to choose an appropriate set of graph statistics. Although it may not be immediately clear what kinds of network properties are relevant, these statistics should match the purpose of the estimation (Hunter et al., 2008). We chose the following set of statistics which we believe represent important aspects of the interbank networks' structure examined by empirical works: mean and coefficient of variation of the degree distribution, density, transitivity, reciprocity, avarege path length, assortativity and mean eigenvector centrality. For instance, we included the mean degree and its coefficient of variation because degree is a fundamental characteristic of the network. We included density to check if the model is able to reproduce the fact that interbank networks are sparse. We added assortativity as an indication of the core-periphery structure in which banks with few connections are generally connected to highly connected banks (Craig and von Peter, 2014). Reciprocal transactions (reciprocity) have a significant effect on the establishment of lending relationships (see, e.g., Affinito, 2012, Bräuning and Fecht, 2016). Transitivity was included because high clustering in financial networks may make banks more vulnerable to contagion effects (Georg, 2013). Average path length is related to the closeness and betweenness measures of centrality and has an effect on the propagation of shocks in the network. Finally, eigenvector centrality has inspired measures of systemic risk as DebtRank (see, e.g., Battiston et al., 2012).

Figure 8 shows kernel density estimates of selected graph statistics for the sim- 

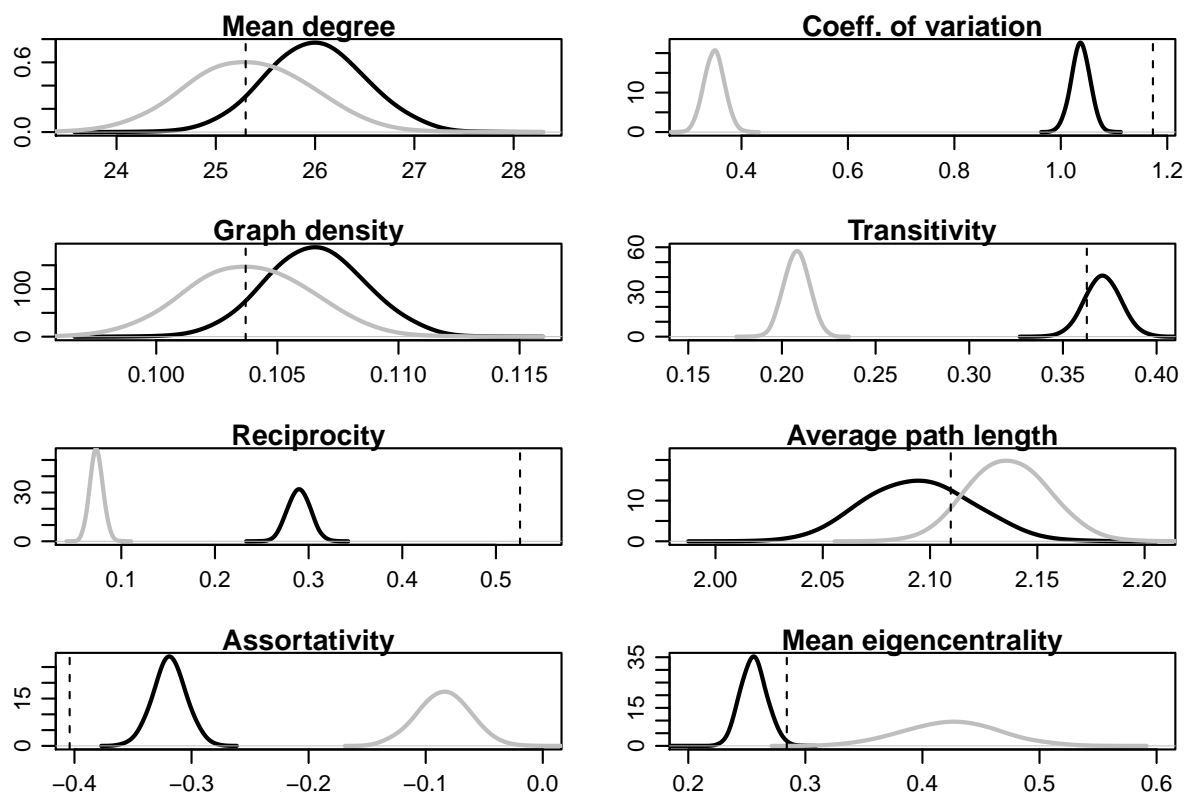

Figure 8: Kernel density estimates of selected graph statistics based on simulated networks under the unsecured network model with the latent space (black line) and without the latent space (gray line). Dashed lines represent the true graph statistics in December, 2016.

ulated data from the model estimated using the unsecured network data. The figure shows simulated data of the model estimated with the latent space (black lines) and without it (gray lines) alongside the observed graph statistics (dashed lines). The figure displays the distributions of graph statistics only for December 2012, although the model accurately replicates graph statistics of the observed networks for the twelve months of data.

According to the density distributions, the inclusion of the latent space improves the model's fit compared to the model incorporating only information on banks' observed characteristics. It is possible to see that the model with the latent space generates datasets $\widetilde{\mathbf{Y}}$ that resemble the observed dataset in terms of different graph statistics of interest such as degree distribution, transitivity and average path length. The model without the latent space is able to generate networks with mean degree similar to the observed data, however the dispersion of the degree distribution is much smaller than the true data. Although for some graph statistics like reciprocity and assortativity the simulated data do not match the observed values, the model which incorporates a latent space fits the data better than the model without the latent space for all graph statistics evaluated, especially for transitivity. 


\section{Conclusion}

In this paper, we have used the dynamic latent space approach of Sewell and Chen (2015) to model directed networks of monthly interbank exposures. In this model, the probability of a tie depends on observed characteristics of a pair of banks and unobserved positions in a low dimension latent space. The model can account for similarity or dissimilarity on observed characteristics as well as interdependence among nodes generally present in network data such as reciprocity and transitivity through the latent space. To model the dynamics of the network it is assumed that the latent node positions follow a Markov process and the observed graph at time $t$ is conditionally independent of all other graphs, given the latent positions and dyadic-level covariate information. Model parameters and latent banks' positions are estimated within a Bayesian framework using a standard Metropolis-Hastings algorithm.

We applied this methodology to analyze two different datasets: the unsecured and the secured (repo) interbank lending networks. Then, we evaluated the fit of a model in which the probability of a tie depends only on observed covariates and a second one in which the latent space is included. This was done by comparing some selected graph statistics calculated for the observed data with distributions obtained from networks simulated under the fitted model. We showed that the model which incorporates a latent space is able to capture some features of the dyadic data such as transitivity. On the other hand, the model in which the probability of forming a tie depends only on observed characteristics of banks has a poor fit. This model is not able to represent the dependencies among ties observed in the network and although the estimated coefficients of both models have same signs, they are very different. Lastly, the inclusion of other covariates in addition to bank's asset size had a small impact on the fit of the model. 


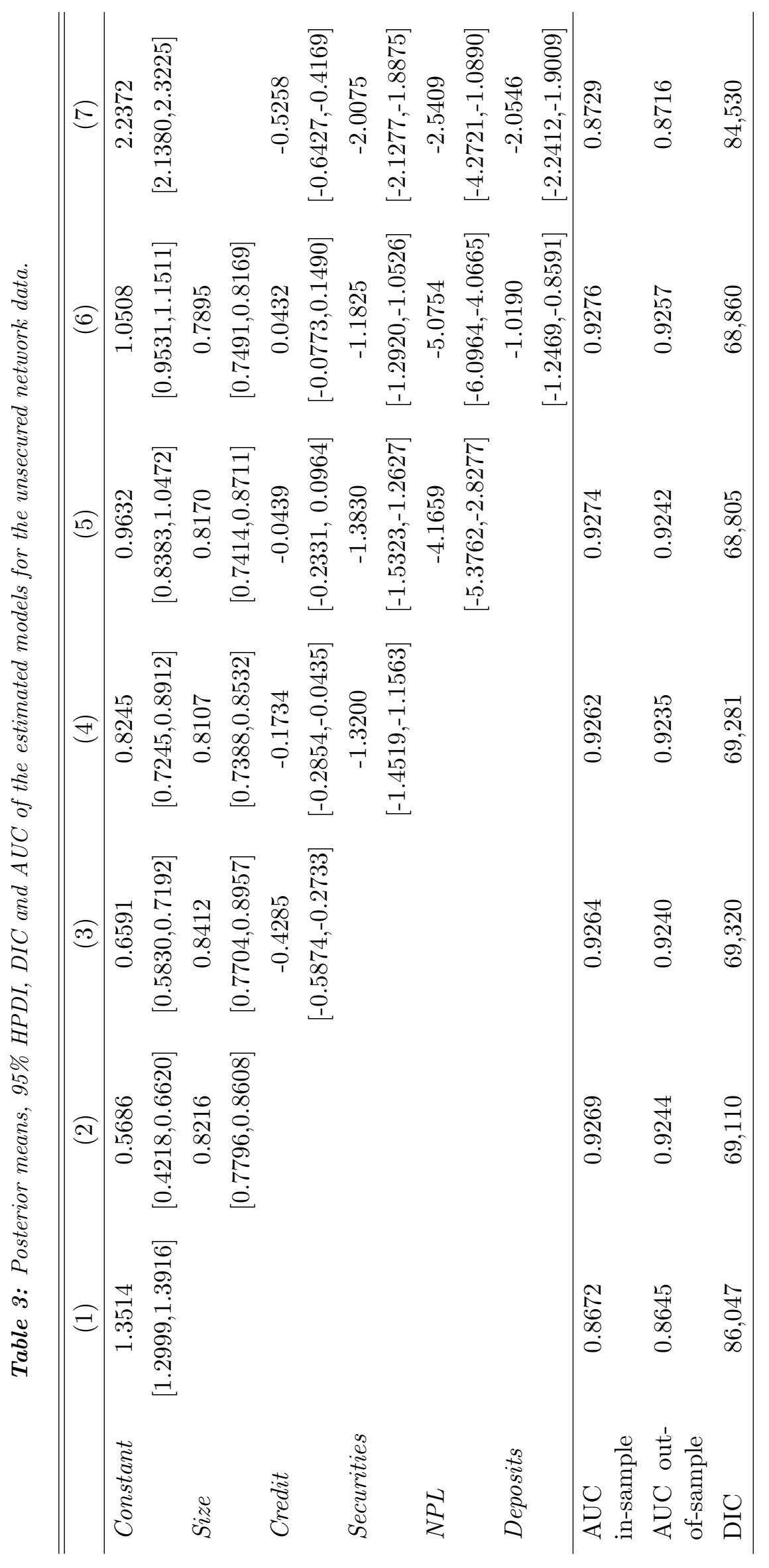




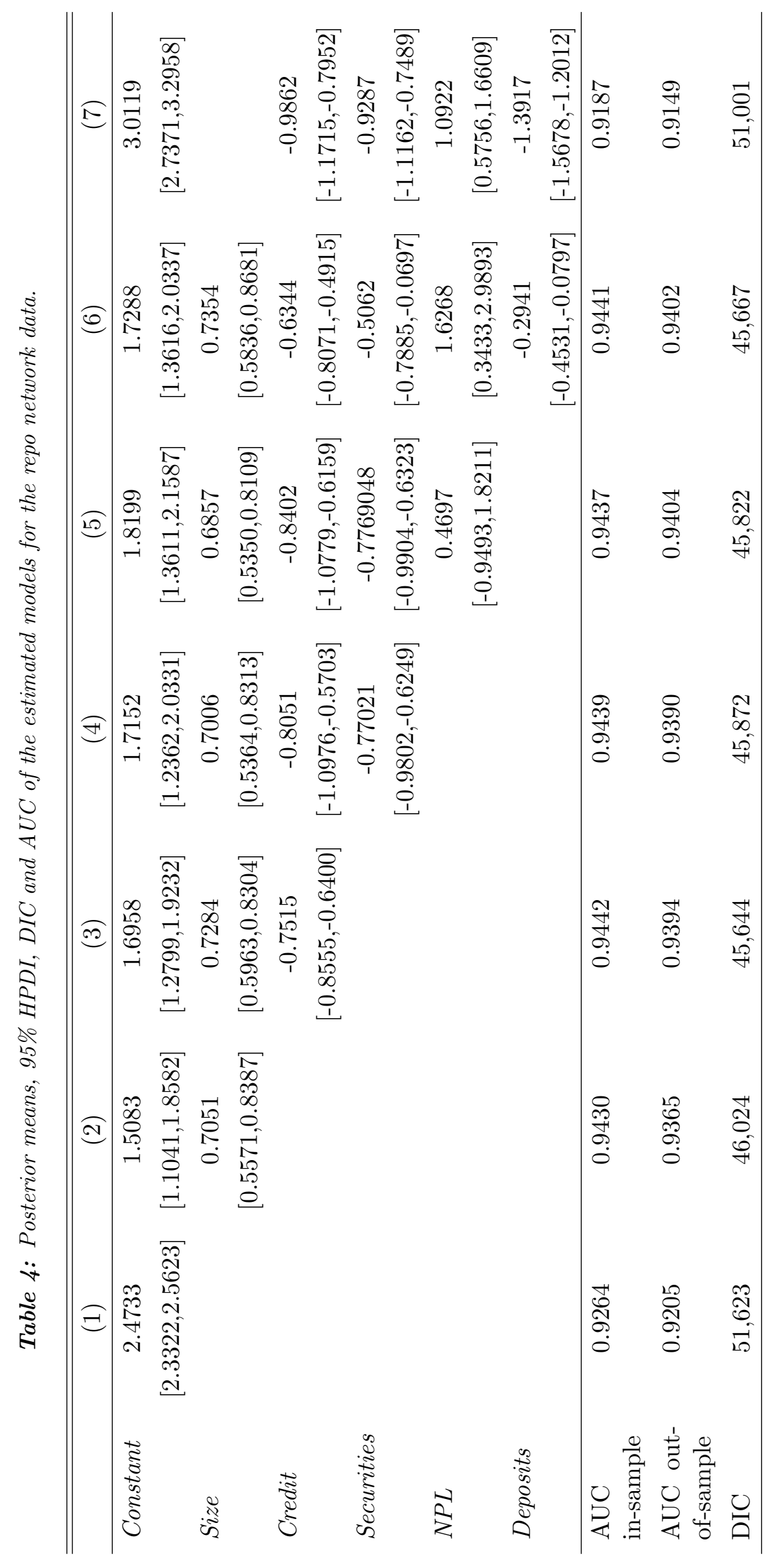




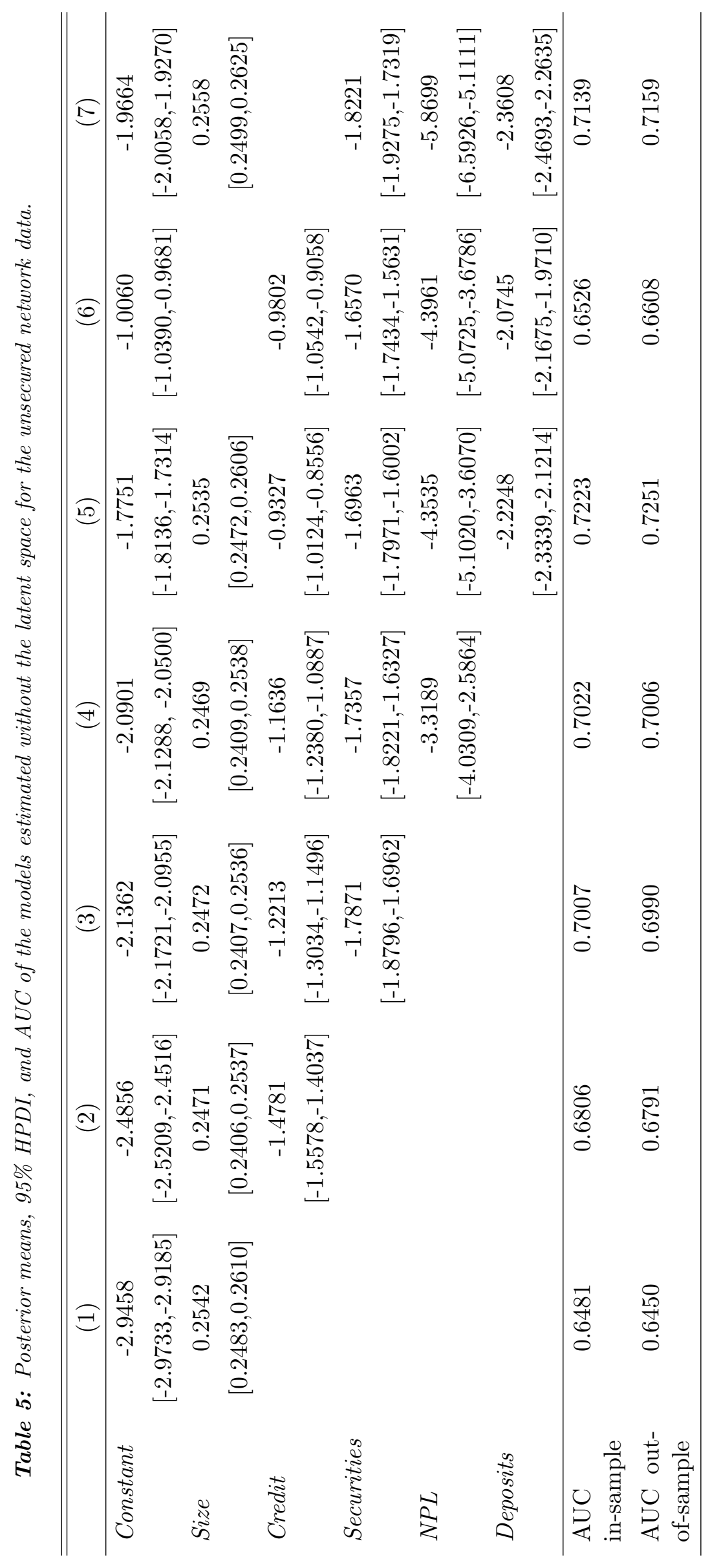




\section{References}

Acemoglu, D., Ozdaglar, A., and Tahbaz-Salehi, A. (2015). Systemic risk and stability in financial networks. American Economic Review, 105(2):564-608.

Affinito, M. (2012). Do interbank customer relationships exist? And how did they function in the crisis? Learning from Italy. Journal of Banking $\&$ Finance, 36(12):3163-3184.

Afonso, G., Kovner, A., and Schoar, A. (2011). Stressed, not frozen: The federal funds market in the financial crisis. Journal of Finance, 66(4):1109-1139.

Afonso, G., Kovner, A., and Schoar, A. (2014). Trading partners in the interbank lending market. Federal Reserve Bank of New York Staff Reports, 620.

Allen, F. and Gale, D. (2000). Financial contagion. Journal of Political Economy, 108(1):1-33.

Battiston, S., Puliga, M., Kaushik, R., Tasca, P., and Caldarelli, G. (2012). DebtRank: Too central to fail? Financial networks, the FED and systemic risk. Scientific Reports, $2(541)$.

Blasques, F., Bräuning, F., and van Lelyveld, I. (2015). A dynamic network model of the unsecured interbank lending market. DNB Working Papers, 460.

Bräuning, F. and Fecht, F. (2016). Relationship lending in the interbank market and the price of liquidity. Review of Finance, 0(0):0.

Cajueiro, D. O. and Tabak, B. M. (2008). The role of banks in the Brazilian interbank market: Does bank type matter? Physica A: Statistical Mechanics and its Applications, 387(27):6825-6836.

Claessens, S. and Kodres, L. (2014). The regulatory responses to the global financial crisis: Some uncomfortable questions. IMF Working Paper, 14/46.

Cocco, J. F., Gomes, F. J., and Martins, N. C. (2009). Lending relationships in the interbank market. Journal of Financial Intermediation, 18(1):24-48.

Cont, R., Santos, E. B., and Moussa, A. (2013). Network structure and systemic risk in banking systems. In Fouque, J. P. and Langsam, J., editors, Handbook of Systemic Risk, pages 327-368. Cambridge University Press. 
Craig, B. and von Peter, G. (2014). Interbank tiering and money center banks. Journal of Financial Intermediation, 23(3):322-347.

Durante, D. and Dunson, D. B. (2014). Nonparametric Bayes dynamic modelling of relational data. Biometrika, 101:883-898.

Durante, D., Dunson, D. B., and Vogelstein, J. T. (2017). Nonparametric Bayes modeling of populations of networks. Journal of the American Statistical Association, $0(0): 0-0$.

Fafchamps, M., Goyal, S., and van der Leij, M. (2010). Matching and network effects. Journal of the European Economic Association, 8(1):203-231.

Finger, K., Fricke, D., and Lux, T. (2013). Network analysis of the e-mid overnight money market: the informational value of different aggregation levels for intrinsic dynamic processes. Computational Management Science, 10(2):187-211.

Freixas, X., Parigi, B. M., and Rochet, J.-C. (2000). Systemic risk, interbank relations, and liquidity provision by the central bank. Journal of Money, Credit, and Banking, 32(3):611-38.

Furfine, C. (1999). The microstructure of the federal funds market. Financial Markets, Institutions, and Instruments, 8(5):24-44.

Gai, P. and Kapadia, S. (2010). Contagion in financial networks. Proceedings of the Royal Society A, 466:2401-23.

Georg, C.-P. (2013). The effect of the interbank network structure on contagion and common shocks. Journal of Banking \&3 Finance, 37(7):2216-28.

Geweke, J. (1992). Evaluating the accuracy of sampling-based approaches to the calculation of posterior moments. In Bayesian Statistics, pages 169-193. University Press.

Geweke, J. and Tanizaki, H. (2001). Bayesian estimation of state-space models using the Metropolis-Hastings algorithm within Gibbs sampling. Computational Statistics E3 Data Analysis, 37(2):151-170.

Giraitis, L., Kapetanios, G., Wetherilt, A., and Zikes, F. (2016). Estimating the dynamics and persistence of financial networks, with an application to the sterling money market. Journal of Applied Econometrics, 31(1):58-84. 
Glasserman, P. and Young, H. P. (2016). Contagion in financial networks. Journal of Economic Literature, 54(3):779-831.

Goldenberg, A., Zheng, A. X., Fienberg, S. E., and Airoldi, E. M. (2009). A survey of statistical network models. Foundations and Trends in Machine Learning, 2(2):1-117.

Goldsmith-Pinkham, P. and Imbens, G. W. (2013). Social networks and the identification of peer effects. Journal of Business 83 Economic Statistics, 31(3):253-262.

Graham, B. S. (2014). Methods of identification in social networks. NBER Working Paper, 20414.

Haario, H., Saksman, E., and Tamminen, J. (2005). Componentwise adaptation for high dimensional MCMC. Computational Statistics, 20(2):265-273.

Hanneke, S., Fu, W., and Xing, E. (2010). Discrete temporal models of social networks. Electronic Journal of Statistics, 4:585-605.

Hoff, P. D. (2011). Hierarchical multilinear models for multiway data. Computational Statistics \& Data Analysis, 55:530-543.

Hoff, P. D., Raftery, A. E., and Handcock, M. S. (2002). Latent space approaches to social network analysis. Journal of the American Statistical Association, 97(460):109098.

Hüser, A.-C. (2015). Too interconnected to fail: A survey of the interbank networks literature. Journal of Network Theory in Finance, 1(3):1-50.

Hunter, D. R., Goodreau, S. M., and Handcock, M. S. (2008). Goodness of fit of social network models. Journal of the American Statistical Association, 103(481):248-258.

Iijima, R. and Kamada, Y. (2016). Social distance and network structures. Theoretical Economics.

Krishnamurthy, A., Nagel, S., and Orlov, D. (2014). Sizing up repo. Journal of Finance, 69(6):2381-2417.

Krivitsky, P. N., Handcock, M. S., Raftery, A. E., and Hoff, P. D. (2009). Representing degree distributions, clustering, and homophily in social networks with latent cluster random effects models. Social Networks, 31(3):204-13. 
Sarkar, P. and Moore, A. W. (2005). Dynamic social network analysis using latent space models. ACM SIGKDD Explorations Newsletter, 7(2):31-40.

Sewell, D. K. and Chen, Y. (2015). Latent space models for dynamic networks. Journal of the American Statistical Association, 110(512):1646-57.

Silva, T. C., de Souza, S. R. S., and Tabak, B. M. (2015). Network structure analysis of the Brazilian interbank market. BCB Working Paper, 391.

Snijders, T. A. B. and Steglich, C. E. G. (2015). Representing micro-macro linkages by actor-based dynamic network models. Sociol Methods Res., 44(2):222-271.

Spiegelhalter, D. J., Best, N. G., Carlin, B. P., and van der Linde, A. (2002). Bayesian measures of model complexity and fit. J. R. Statist. Soc. B, 64(4):583-639.

Squartini, T., van Lelyveld, I., and Garlaschelli, D. (2013). Early-warning signals of topological collapse in interbank networks. Scientific reports, 3(3357).

Tabak, B. M., Takami, M., Rocha, J. M., Cajueiro, D. O., and Souza, S. R. (2014). Directed clustering coefficient as a measure of systemic risk in complex banking networks. Physica A: Statistical Mechanics and its Applications, 394:211-216.

Xing, E. P., Fu, W., and Song, L. (2010). A state-space mixed membership blockmodel for dynamic network tomography. The Annals of Applied Statistics, 4(2):535-566.

\section{A Appendix: Posterior distributions}

We derive the posterior density of $\mathbf{Z}_{t}$ for the Bayesian estimation of state-space models using Gibbs sampler and MH algorithm. See Geweke and Tanizaki (2001) and Sewell and Chen (2015) for more details. Considering the state-space model of Section 2, the density of $\mathbf{Z}_{1: T}$ and $\mathbf{Y}_{1: T}$ given $\boldsymbol{\psi}=\left(\tau^{2}, \sigma^{2}\right), \boldsymbol{\beta}$ and $\mathbf{X}_{1: T}$ is written as:

$$
\pi\left(\mathbf{Z}_{1: T}, \mathbf{Y}_{1: T} \mid \mathbf{X}_{1: T}, \boldsymbol{\psi}, \boldsymbol{\beta}\right)=\pi\left(\mathbf{Z}_{1: T} \mid \boldsymbol{\psi}\right) \pi\left(\mathbf{Y}_{1: T} \mid \mathbf{Z}_{1: T}, \mathbf{X}_{1: T}, \boldsymbol{\beta}\right)
$$

where the two densities in the right hand side are represented by:

$$
\pi\left(\mathbf{Z}_{1: T} \mid \boldsymbol{\psi}\right)=\prod_{t=1}^{T} \pi\left(\mathbf{Z}_{t} \mid \mathbf{Z}_{(t-1)}, \boldsymbol{\psi}\right)
$$




$$
\pi\left(\mathbf{Y}_{1: T} \mid \mathbf{Z}_{1: T}, \mathbf{X}_{1: T}, \boldsymbol{\beta}\right)=\prod_{t=1}^{T} \pi\left(\mathbf{Y}_{t} \mid \mathbf{Z}_{t}, \mathbf{X}_{1: T}, \boldsymbol{\beta}\right)
$$

The conditional distribution of $\mathbf{Z}_{1: T}$ is

$$
\pi\left(\mathbf{Z}_{1: T} \mid \mathbf{Y}_{1: T}, \mathbf{X}_{1: T}, \boldsymbol{\psi}, \boldsymbol{\beta}\right)=\frac{\pi\left(\mathbf{Z}_{1: T}, \mathbf{Y}_{1: T} \mid \mathbf{X}_{1: T}, \boldsymbol{\psi}, \boldsymbol{\beta}\right)}{\int \pi\left(\mathbf{Z}_{1: T}, \mathbf{Y}_{1: T} \mid \mathbf{X}_{1: T}, \boldsymbol{\psi}, \boldsymbol{\beta}\right) d \mathbf{Z}_{1: t}}
$$

The conditional density function of $\mathbf{Z}_{t}$ given $\mathbf{Z}_{1: t-1}, \mathbf{Z}_{t+1: T}, \mathbf{X}_{1: T}, \boldsymbol{\psi}$ and $\boldsymbol{\beta}$ is derived from the previous equations and is shown below:

$$
\begin{aligned}
& \pi\left(\mathbf{Z}_{t} \mid \mathbf{Z}_{1: t-1}, \mathbf{Z}_{t+1: T}, \mathbf{Y}_{1: T}, \mathbf{X}_{1: T}, \boldsymbol{\psi}, \boldsymbol{\beta}\right)=\frac{\pi\left(\mathbf{Z}_{1: T} \mid \mathbf{Y}_{1: T}, \mathbf{X}_{1: T}, \boldsymbol{\psi}, \boldsymbol{\beta}\right)}{\int \pi\left(\mathbf{Z}_{1: T} \mid \mathbf{Y}_{1: T}, \mathbf{X}_{1: T}, \boldsymbol{\psi}, \boldsymbol{\beta}\right) d \mathbf{Z}_{t}} \\
& \propto\left\{\begin{array}{cc}
\pi\left(\mathbf{Y}_{t} \mid \mathbf{Z}_{t}, \mathbf{X}_{1: T}, \boldsymbol{\beta}\right) \pi\left(\mathbf{Z}_{t} \mid \mathbf{Z}_{t-1}, \boldsymbol{\psi}\right) \pi\left(\mathbf{Z}_{t+1} \mid \mathbf{Z}_{t}, \boldsymbol{\psi}\right) & \text { if } t=1, \ldots T-1 \\
\pi\left(\mathbf{Y}_{t} \mid \mathbf{Z}_{t}, \mathbf{X}_{1: T}, \boldsymbol{\beta}\right) \pi\left(\mathbf{Z}_{t} \mid \mathbf{Z}_{t-1}, \boldsymbol{\psi}\right) & \text { if } t=T
\end{array}\right.
\end{aligned}
$$

Equation 14 gives the conditional distribution of $\mathbf{Z}_{t}$. The conditional distribution of the row $\mathbf{z}_{i t}$ is proportional to:

$$
\pi\left(\mathbf{z}_{i t} \mid \mathbf{Y}_{1: t}, \mathbf{z}_{1: t}, \mathbf{X}_{1: t}, \boldsymbol{\psi}, \boldsymbol{\beta}\right) \propto\left\{\begin{array}{cc}
\left(\prod_{j: j \neq i} p_{i j t} p_{j i t}\right) \mathrm{N}\left(\mathbf{z}_{i t} \mid \mathbf{0}, \tau^{2} \mathbf{I}_{p}\right) \mathrm{N}\left(\mathbf{z}_{i(t+1)} \mid \mathbf{z}_{i t}, \sigma^{2} \mathbf{I}_{p}\right) & \text { if } t=1 \\
\left(\prod_{j: j \neq i} p_{i j t} p_{j i t}\right) \mathrm{N}\left(\mathbf{z}_{i(t+1)} \mid \mathbf{z}_{i t}, \sigma^{2} \mathbf{I}_{p}\right) \mathrm{N}\left(\mathbf{z}_{i t} \mid \mathbf{z}_{i(t-1)}, \sigma^{2} \mathbf{I}_{p}\right) & \text { if } 1<t<T \\
\left(\prod_{j: j \neq i} p_{i j t} p_{j i t}\right) \mathrm{N}\left(\mathbf{z}_{i t} \mid \mathbf{z}_{i(t-1)}, \sigma^{2} \mathbf{I}_{p}\right) & \text { if } t=T
\end{array}\right.
$$

where $p_{i j t} \doteq \mathbb{P}\left(y_{i j t}=1 \mid \mathbf{Z}_{t}, \mathbf{X}_{t}, \boldsymbol{\beta}\right)$ and $\boldsymbol{\psi}=\left(\tau^{2}, \sigma^{2}\right)$. The posterior density of the model parameters $\boldsymbol{\beta}^{\prime}$ follows the form:

$$
\pi\left(\boldsymbol{\beta} \mid \mathbf{Y}_{1: T}, \mathbf{Z}_{1: T}, \mathbf{X}_{1: T}\right) \propto\left[\prod_{t=1}^{T} \prod_{i \neq j} p_{i j t}\right] \cdot \operatorname{MVN}_{d}\left(\boldsymbol{\beta} \mid \boldsymbol{\mu}_{\beta}, \boldsymbol{\Sigma}_{\beta}\right) .
$$

The parameters $\tau^{2}$ and $\sigma^{2}$ can be Gibbs-sampled and their full conditional distributions are as follows:

$$
\begin{gathered}
\pi\left(\tau^{2} \mid \mathbf{Z}_{1}\right) \sim \operatorname{IG}\left(\left(\nu_{\tau}+n p\right) / 2, \nu_{\tau} \xi_{\tau}^{2} / 2+\frac{1}{2} \sum_{i=1}^{n}\left\|\mathbf{z}_{i 1}\right\|^{2}\right) \\
\pi\left(\sigma^{2} \mid \mathbf{Z}_{1: T}\right) \sim \operatorname{IG}\left(\left(\nu_{\sigma}+n p(T-1)\right) / 2, \nu_{\sigma} \xi_{\sigma}^{2} / 2+\frac{1}{2} \sum_{t=2}^{T} \sum_{i=1}^{n}\left\|\mathbf{z}_{i t}-\mathbf{z}_{i(t-1)}\right\|^{2}\right)
\end{gathered}
$$




\section{B Appendix: Initialization}

The starting values of the Markov chain are obtained as follows:

1. Latent positions $\mathbf{Z}_{1: T}$ : Hoff et al. (2002) use multidimensional scaling (MDS) to get the initial estimates of the latent positions. The MDS takes the $n \times n$ matrix of distances between objects and returns their positions in a lower dimensional space for visualization while preserving the distances as well as possible. Sarkar and Moore (2005) extend the MDS by using it to get the initial estimates of $\mathbf{Z}_{1}^{(1)}$. Then, for $t=2, \ldots, T$, they perform the MDS along with the objective of minimizing $\left|\mathbf{Z}_{t}^{(1)}-\mathbf{Z}_{t-1}^{(1)}\right|_{F}$, where $|\cdot|_{F}$ denotes the Frobenius norm, to avoid that the node positions change drastically from one time step to another. For the matrices of distances, Sewell and Chen (2015) suggest the use of the length of the shortest path from $i$ to $j$ at time $t$ scaled by $1 / n$. We use to different methods to obtain a set of dissimilarity measures between pairs of banks: the shortest path length and the absolute value of the difference of the $\log$ of bank assets.

2. Parameters $\tau^{2}$ and $\sigma^{2}$ : The initial value for $\tau^{2}$ was set using the initial estimates of $\mathbf{Z}_{1}$ and is given by:

$$
\frac{1}{n p} \sum_{i=1}^{n}\left\|\mathbf{z}_{i 1}\right\|^{2}
$$

It is possible to obtain initial estimate of $\sigma^{2}$ using the same procedure, but Sewell and Chen (2015) recommend to set a large initial value for it to allow large movements of the latent positions at the beginning of iterations and increase the speed of convergence.

3. Parameter $\boldsymbol{\beta}$ : Using the initial estimates of the latent positions, starting value of $\boldsymbol{\beta}$ is found by numerical maximization of the observation equation (optim function of the $\mathrm{R}$ environment).

\section{Appendix: Network statistics}

In this appendix we describe the network statistics that are used in the paper. The density of a network is the ratio of the number of existing edges to the number of possible edges. It is given by:

$$
\operatorname{Den}\left(\mathcal{G}_{t}\right)=\sum_{i, j} \frac{y_{i j t}}{n(n-1)} .
$$


Transitivity or clustering coefficient is the probability that adjacent nodes of a node are also connected:

$$
C l\left(\mathcal{G}_{t}\right)=\frac{\sum_{i, j \neq i, k \neq j, k \neq i} \widetilde{y}_{i j t} \widetilde{y}_{i k t} \widetilde{y}_{j k t}}{\sum_{i, j \neq i, k \neq j, k \neq i} \widetilde{y}_{i j t} \widetilde{y}_{i k t}}
$$

where $\widetilde{y}_{i j t}=\max \left\{y_{i j t}, y_{j i t}\right\}$. In this case, transitivity is computed ignoring the direction of the edges. Reciprocity is the proportion of the total number of reciprocated edges to the total number of edges:

$$
\operatorname{Re}\left(\mathcal{G}_{t}\right)=\frac{\sum_{i, j} y_{i j t} y_{j i t}}{\sum_{i, j} y_{i j t}} .
$$

Transitivity can also be calculated for each node, and then averaging across all nodes. Reciprocity can also be computed as the number of reciprocated dyads divided by the number of not connected or single connected dyads.

The (geodesic) distance between nodes $i$ and $j$ in a network is the length of the shortest path between them. This distance, which will be denoted by $d\left(i, j ; \mathcal{G}_{t}\right)$, is used to compute the average path length and the diameter. These two measures are computed considering only directed paths. The average path length is the average length of the shortest paths between nodes:

$$
d\left(\mathcal{G}_{t}\right)=\frac{\sum_{i, j} d\left(i, j ; \mathcal{G}_{t}\right)}{n(n-1)}
$$

while the diameter is the length of the longest geodesic in a graph:

$$
D\left(\mathcal{G}_{t}\right)=\max _{i, j} d\left(i, j ; \mathcal{G}_{t}\right)
$$

